Article

\title{
Fabrication and Optimization of Bilayered Nanoporous Anodic Alumina Structures as Multi-Point Interferometric Sensing Platform
}

\author{
Mahdieh Nemati ${ }^{1}$, Abel Santos ${ }^{1,2,3}$ and Dusan Losic ${ }^{1, *(1)}$ \\ 1 School of Chemical Engineering, The University of Adelaide, Engineering North Building, Adelaide 5005, \\ Australia; mahdieh.nemati@adelaide.edu.au (M.N.); abel.santos@adelaide.edu.au (A.S.) \\ 2 Institute for Photonics and Advanced Sensing (IPAS), The University of Adelaide, Adelaide 5005, Australia \\ 3 ARC Centre of Excellence for Nanoscale BioPhotonics (CNBP), The University of Adelaide, \\ Adelaide 5005, Australia \\ * Correspondence: dusan.losic@adelaide.edu.au; Tel.: +61-8-8313-4648
}

Received: 19 December 2017; Accepted: 31 January 2018; Published: 6 February 2018

\begin{abstract}
Herein, we present an innovative strategy for optimizing hierarchical structures of nanoporous anodic alumina (NAA) to advance their optical sensing performance toward multi-analyte biosensing. This approach is based on the fabrication of multilayered NAA and the formation of differential effective medium of their structure by controlling three fabrication parameters (i.e., anodization steps, anodization time, and pore widening time). The rationale of the proposed concept is that interferometric bilayered NAA (BL-NAA), which features two layers of different pore diameters, can provide distinct reflectometric interference spectroscopy (RIfS) signatures for each layer within the NAA structure and can therefore potentially be used for multi-point biosensing. This paper presents the structural fabrication of layered NAA structures, and the optimization and evaluation of their RIfS optical sensing performance through changes in the effective optical thickness (EOT) using quercetin as a model molecule. The bilayered or funnel-like NAA structures were designed with the aim of characterizing the sensitivity of both layers of quercetin molecules using RIfS and exploring the potential of these photonic structures, featuring different pore diameters, for simultaneous size-exclusion and multi-analyte optical biosensing. The sensing performance of the prepared NAA platforms was examined by real-time screening of binding reactions between human serum albumin (HSA)-modified NAA (i.e., sensing element) and quercetin (i.e., analyte). BL-NAAs display a complex optical interference spectrum, which can be resolved by fast Fourier transform (FFT) to monitor the EOT changes, where three distinctive peaks were revealed corresponding to the top, bottom, and total layer within the BL-NAA structures. The spectral shifts of these three characteristic peaks were used as sensing signals to monitor the binding events in each NAA pore in real-time upon exposure to different concentrations of quercetin. The multi-point sensing performance of BL-NAAs was determined for each pore layer, with an average sensitivity and low limit of detection of $600 \mathrm{~nm}\left(\mathrm{mg} \mathrm{mL}^{-1}\right)^{-1}$ and $0.14 \mathrm{mg} \mathrm{mL}^{-1}$, respectively. BL-NAAs photonic structures have the capability to be used as platforms for multi-point RIfS sensing of biomolecules that can be further extended for simultaneous size-exclusion separation and multi-analyte sensing using these bilayered nanostructures.
\end{abstract}

Keywords: nanoporous anodic alumina; structural fabrication; reflectometric interference spectroscopy; optical sensors; biomolecule detection 


\section{Introduction}

There are an increasing number of research studies focused on exploring new nanoporous materials and their applications in highly sensitive chemical and biosensing devices which are cost-effective and more sensitive due to their specific surface areas and the unique properties of their pore structures. Among them, nanoporous anodic alumina (NAA) fabricated by electrochemical anodization has been extensively explored for optical transduction systems in a broad range of sensing applications including biomedical, pharmaceutical, industrial, and environmental [1]. The versatile nanoporous structure of NAA makes it an excellent optical platform to develop sensing devices based on broad ranges of detection principles including UV-Vis, surface plasmon resonance, reflective interference, optical waveguiding, Raman spectroscopy and others [2-4]. NAA pore structures are highly sensitive to any alteration of the effective medium including the binding of molecules, which can be subsequently translated into readable optical signals [5-8]. Reflectometric interference spectroscopy (RIfS) is one of most attractive optical sensing techniques used for development of low cost and simple sensing devices [9-12]. The method is based on interaction of optical platforms and light reflection where reflected light is amplified at specific wavelengths with enhanced reflections, creating characteristic interferometric spectra due to the Fabry-Pérot effect. In NAAs, the Fabry-Pérot effect can be described by Equation (1),

$$
\mathrm{EOT}=2 \mathrm{n}_{\mathrm{eff}} \mathrm{L} \cos \theta=m \lambda,
$$

where EOT is the effective optical thickness of the NAA film, $n_{\text {eff }}$ is its effective refractive index, $\mathrm{L}$ is its physical thickness, $m$ is the order of wavelength oscillation in the spectrum of RIfS, $\lambda$ is maximum wavelength, and $\theta$ is the incidence angle of light. This expression can be used to quantify changes in the effective medium of the NAA platform, using effective optical thickness changes $(\triangle \mathrm{EOT})$ as the sensing parameter [13]. RIfS using optical platforms were extensively explored by the Gauglitz group and others to detect a broad range of analytes including organic molecules, gases, DNA, pesticides, etc. [14-17].

In contrast to RIfS, systems based on solid optical platforms, the combination of RIfS with nanoporous platforms such as NAA has a set of attractive properties such as a high effective surface area which increases the number of binding centres and effective medium that can be fabricated with precision to create NAA structures with highly sensitive sensing platforms $[1,18]$. Our previous studies demonstrated that the NAA can be successfully used for the development of highly sensitive RIfS devices for a broad range of applications including organic molecules, enzymes, drugs, cancer cells, etc. [13,19-22]. To advance sensing and optical (RIfS) performance of these NAA platforms many strategies have been employed using structural fabrication methods to optimise pore structures, introduce appropriate surface modifications and design of RIfS sensing devices combining with microfluidics $[23,24]$. Several studies have demonstrated that the nanoporous structure of NAA can be precisely fabricated by different anodization approaches to generate multi-dimensional photonic crystal structures such as distributed Bragg reflectors and funnel-like photonic films that can significantly improve their sensing performance $[25,26]$. Marshal's group and others recently demonstrated that funnel-like NAA structures (multi-layered NAA films featuring a decreasing pore diameter from top to bottom) can provide characteristic RIfS properties that could offer new sensing applications [27-30]. It is indicated that bilayered NAA (BL-NAA) nanostructures feature a complex RIfS spectrum, which can be resolved by Fourier transform (FFT) to discern characteristic peaks associated with each layer and be independently utilized to monitor and quantify molecular binding events occurring in each layer within the BL-NAA structure [31-33]. However, more fundamental and applied studies are needed in order to fully understand RIfS characteristics of bi-layered and multi-layered NAA structures and exploit their potential for multi-point and multi sensing applications.

In this study, we fabricated bi-layered NAA films (BL-NAA) with hierarchical funnel-like structures in order to explore their RIfS performances and potential to be used for advance biosensing, including multi-analyte detection. Our fabrication approach consisted of several sequential 
anodization and chemical etching steps, which enable the design of bi-layered optical structures with a specific pore diameter and length in depth. It is proposed that fabricated BL-NAA structures will feature three distinct and well-resolved peaks in their FFT spectrum that correspond to each physical layer (top layer I, bottom layer II, and total layer III). These characteristic peaks are proposed for independent molecular sensing inside pores in order to explore their simultaneous size-exclusion and multi analytes detection. Quercetin is a plant flavonoid present in various fruits, vegetables and medicinal herbs [34] that has as antioxidant [35,36] properties with the potential for therapeutic applications such as neuroprotective effect, cardiovascular protection, anti-cancer, and anti-inflammatory properties [37-39]. To achieve selectivity, the surface chemistry was functionalized with human serum albumin (HSA) attached on 3-aminopropyl) trimethoxysilane (APTES) in order to endow these with chemical selectivity towards polyphenol quercetin. Binding events between quercetin and HSA molecules are measured in real-time measurements through changes of effective optical thickness (EOT) of each layer in the FFT spectrum of BL-NAA by reflective interferometric spectroscopy (RIfS), enabling the assessment of the optical sensing performance of BL-NAAs platform. A systematic study analysing the effect of the geometric features of BL-NAA on the sensitivity and low limit of detection was performed in order to determine the limitations and potential of this system for multi-analyte and size-exclusion biosensing.

\section{Materials and Methods}

\subsection{Materials}

High purity (99.9997\%) aluminum foils (Al) $0.32 \mathrm{~mm}$ thick were supplied by Goodfellow Cambridge Ltd. (Huntingdon, UK). Oxalic acid $\left(\mathrm{C}_{2} \mathrm{H}_{2} \mathrm{O}_{4}\right)$, perchloric acid $\left(\mathrm{HClO}_{4}\right)$, phosphoric acid $\left(\mathrm{H}_{3} \mathrm{PO}_{4}\right)$, chromic acid $\left(\mathrm{H}_{2} \mathrm{CrO}_{4}\right)$, (3-aminopropyl)trimethoxysilane (APTES), hydrogen peroxide $\left(\mathrm{H}_{2} \mathrm{O}_{2}\right)$, glutaraldehyde $\left(\mathrm{CH}_{2}\left(\mathrm{CH}_{2} \mathrm{CHO}\right)_{2}-\mathrm{GTA}\right)$, sodium hydroxide $(\mathrm{NaOH})$, human serum albumin (HSA), quercetin $\left(\mathrm{C}_{15} \mathrm{H}_{10} \mathrm{O}_{7}\right)$, and phosphate buffer saline (PBS) were purchased from Sigma-Aldrich (Australia). Ultrapure water from Option Q-Purelabs (Australia) was used for preparing the aqueous solutions used in this study.

\subsection{Fabrication of Bilayered NAA Films (BL-NAAs) and NAAs}

Al samples were anodized in an electrochemical cell by a multiple step anodization process. $\mathrm{Al}$ samples were first sonicated in ethanol to remove organic residue and electrochemically polished in a mixture of EtOH: $\mathrm{HClO}_{4}$ 4:1 (v:v) at $20 \mathrm{~V}$ and $5{ }^{\circ} \mathrm{C}$ for $3 \mathrm{~min}$ to achieve a smooth surface. The first anodization step was performed in a solution of $0.3 \mathrm{M} \mathrm{H}_{2} \mathrm{C}_{2} \mathrm{O}_{4}$ at $40 \mathrm{~V}$ and $5{ }^{\circ} \mathrm{C}$ for $20 \mathrm{~h}$. After that, the NAA structure was etched in an aqueous solution of $0.2 \mathrm{M} \mathrm{H}_{2} \mathrm{CrO}_{4}$ and $0.4 \mathrm{M} \mathrm{H}_{3} \mathrm{PO}_{4}$ at $70{ }^{\circ} \mathrm{C}$ for $3 \mathrm{~h}$. Two anodization steps were carried out using $0.3 \mathrm{M} \mathrm{H}_{2} \mathrm{C}_{2} \mathrm{O}_{4}$ at $40 \mathrm{~V}$ and $5{ }^{\circ} \mathrm{C}$ for different duration. The fabrication protocol for each BL-NAA platform is detailed in Table 1. A pore widening treatment between anodization steps was used to increase the diameter of the nanopores. This process was carried out by wet chemical etching in an aqueous solution of $\mathrm{H}_{3} \mathrm{PO}_{4} 5 \mathrm{wt}$. $\%$ at $35^{\circ} \mathrm{C}$ for $15 \mathrm{~min}$ (Table 1 ). Figure 1 shows a generic illustration of the fabrication process used to produce BL-NAA. We fabricated two control NAAs wer under the same anodization conditions featuring straight nanopores from top to bottom at 0 and 15 in of pore widening treatment (Table 1 ). 


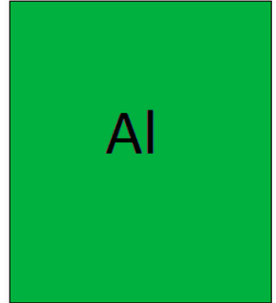

(a)

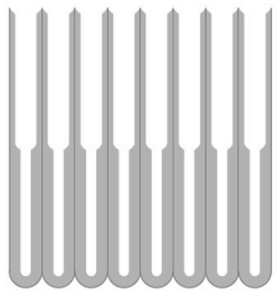

(f)

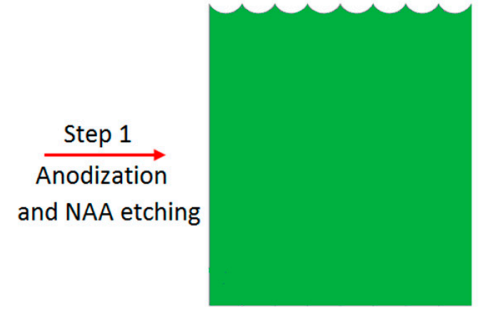

(b)

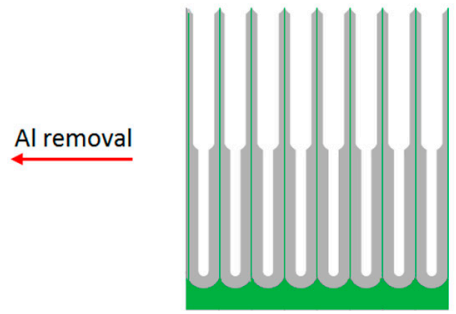

(e)
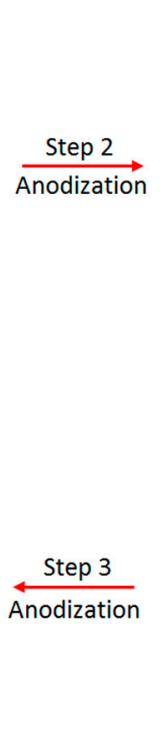

Figure 1. Fabrication process of interferometric bilayered nanoporous anodic alumina (BL-NAA) structures. (a) Electro polished high purity aluminum; (b) First step of anodization and resulting chemically etched nanostructure; (c) Second step of anodization results top layer of BL-NAAs; (d) Pore-widening leads to nanopores with a larger pore diameter; (e) Third step of anodization results in the bottom layer of the nanopore structure which has a smaller pore diameter compared to top layer; (f) Supporting aluminum was removed from the backside of platform. * Note that, stage (e) was not included for nanoporous anodic alumina (NAAs) fabrication.

Table 1. Fabrication protocol of BL-NAAs and NAAs that combines anodization and pore widening steps BL-NAA $_{(25 / 75)}$ represent ratio in lengths of pore layers with $25 \%$ top layer with larger pore diameters and $75 \%$ of layer with layer with smaller pore diameters, BL-NAA $_{(50 / 50)}$ with equal length of both layers and the BL-NAA $(75 / 25)$ structure with $75 \%$ top layer with larger pore diameters and $25 \%$ of layer with layer with smaller pore diameters, and $\mathrm{NAA}_{(1)}$ with uniform structures with large pore diameters and $\mathrm{NAA}_{(2)}$ with smaller pore diameters.)

\begin{tabular}{|c|c|c|c|c|c|}
\hline \multicolumn{2}{|c|}{$\begin{array}{c}\text { Anodized Anodic Aluminum } \\
\text { Platforms }\end{array}$} & \multirow{2}{*}{$\begin{array}{c}\begin{array}{c}\text { Step (1) } \\
\text { Anodization (h) }\end{array} \\
20\end{array}$} & \multirow{2}{*}{$\begin{array}{c}\text { Step (2) } \\
\text { Anodization (h) } \\
3\end{array}$} & \multirow{2}{*}{$\begin{array}{c}\begin{array}{c}\text { Pore Widening } \\
\text { (min) }\end{array} \\
15\end{array}$} & \multirow{2}{*}{$\begin{array}{c}\text { Step (3) } \\
\text { Anodization (h) } \\
6\end{array}$} \\
\hline \multirow{2}{*}{ NAAF } & $\mathrm{BL}^{-N A A}(25 / 75)$ & & & & \\
\hline & $\mathrm{BL}^{-N A A}(75 / 25)$ & 20 & 6 & 15 & 3 \\
\hline NAA & $\mathrm{NAA}_{(1)}$ & 20 & 9 & 15 & $\mathrm{~N} / \mathrm{A}^{1}$ \\
\hline
\end{tabular}

${ }^{1}$ N/A: Not applied.

\subsection{Surface Chemistry Functionalization of BL-NAAs and NAAs}

The fabricated BL-NAAs and NAAs were chemically functionalized with APTES following a well-established protocol $[1,40]$. In brief, hydroxyl groups were created on the inner surface of BL-NAAs and NAAs by immersion in $30 \mathrm{wt} . \% \mathrm{H}_{2} \mathrm{O}_{2}$ at $90{ }^{\circ} \mathrm{C}$ over $15 \mathrm{~min}$. After that, silane molecules were immobilized onto the inner surface of BL-NAA nanopores by chemical vapor deposition method. This process was carried out under vacuum condition at $135^{\circ} \mathrm{C}$ for $3 \mathrm{~h}$. Finally, BL-NAAs and NAAs were coated with an ultrathin gold film (i.e., $5 \mathrm{~nm}$ ) deposited by a sputter coater (sputter coater 108auto, Cressington, Redding, CA, USA) in order to enhance the light interference as reported elsewhere [41]. 


\subsection{Optical Sensitivity Assessment of BL-NAAs and NAAs by RIfS}

The sensing performance of BL-NAAs and NAAs was assessed by measuring changes of the EOT in each optical layer using quercetin as a sensing agent model to establish the sensing parameters including sensitivity $(S)$, linearity $\left(R^{2}\right)$, and low limit of detection (LLOD). These sensing parameters were estimated by measuring shifts in the EOT of each layer according to Equation (1).

Real-time screening of EOT shifts was performed using a RIfS system combined with a cell flow system. The APTES functionalized BL-NAAs and NAAs were placed into the flow cell, where phosphate buffer saline (PBS) was allowed to flow until a stable baseline was achieved. In order to immobilize HSA onto the surface, $2.5 \%$ glutaraldehyde was used as a cross-linker and flowed through the system for $30 \mathrm{~min}$ over BL-NAAs and NAAs. GTA molecules activate the amine functional group $\left(-\mathrm{NH}_{2}\right)$ of APTES molecules inside nanopores of BL-NAAs and NAAs.

PBS was flowed again for $15 \mathrm{~min}$ to wash non-covalent bound of GTA molecules. After this, $1 \mathrm{mg} \mathrm{mL}^{-1}$ human serum albumin (HSA) solution was allowed to flow through the system for $1.5 \mathrm{~h}$ in order to immobilize HSA onto the inner surface of BL-NAA and NAAs. Next, PBS solution was allowed to flow for $15 \mathrm{~min}$ to remove physiosorbed HSA molecules. After that, analytical solutions of the analyte agent, quercetin, with different concentrations (i.e., $0.05,0.125,0.25,0.375,0.5,1 \mathrm{mM}$ ) were allowed to flow through the system untill a stable line was achieved confirming that all HSA molecules were saturated with quercetin. Finally, PBS solution was let flow to establish the total EOT changes associated to HSA-Quercetin binding. Note that, this process was carried out at $\mathrm{pH} 7.5$ and room temperature.

\subsection{Structural Characterization of BL-NAAF and NAAs}

The structural characteristics of the prepared BL-NAAs and NAAs (top and fractured structures) were established by field emission scanning electron microscopy (FEG-SEM FEI Quanta 450, ThermoFisher Scientific, OR, USA). Imaging was performed at least on four different spots for each sample in order to probe the reproducibility of process and uniformity of the fabricated structures.

\section{Results and Discussion}

\subsection{Structural Characterisation of Prepared BL-NAAs and NAAs}

The morphology of BL-NAAs prepared using different anodization conditions to make materials with different thicknesses and pore diameters of each layer and control NAAs structures (with single pore layer) characterized by SEM are presented in Figure 2 for BL-NAAs and in the supplementary information for the control NAAs structures. A representative top view SEM image of BL-NAAs confirmed the same pore shape and uniform pore size on the surface for BL-NAA $(25 / 75), \mathrm{BL}-\mathrm{NAA}_{(50 / 50)}$, $\operatorname{BL}_{-N A A}(75 / 25), \mathrm{NAA}_{(1)}$, and NAA $(2)$ (supplementary information). Table 2 shows the overall pore dimensions of BL-NAAs and NAAs structures. The pore diameters of the top layer for BL-NAA $(25 / 75)$, BL-NAA $_{(50 / 50)}$, BL-NAA $_{(75 / 25)}, \mathrm{NAA}_{(1)}$ generated by anodization and pore widening process was confirmed to be about $55 \mathrm{~nm}$ and similar for all samples. The pore diameters of $\mathrm{NAA}_{(2)}$ prepared only by the anodization process are $45 \mathrm{~nm}$ in size and the bottom layer pore sizes were confirmed by cross-sectional imaging. Cross-sectional images of BL-NAA revealed two stack layers of cylindrical and vertical ordered nanostructures, the top layer with larger and the bottom layer with smaller pore dimeters was confirmed by a series of SEM images (Figure 2f). The series of cross section images with different magnifications showed three different types of bi-layered structures with thicknesses of $30 \pm 1.5$ to $32 \pm 1.6 \mu \mathrm{m}$ and different length of top and bottom layers. The length of the top nanoporous layer was $9 \pm 0.45 \mu \mathrm{m}$ for BL-NAA $(25 / 75), 15 \pm 0.75 \mu \mathrm{m}$ for BL-NAA ${ }_{(50 / 50)}$ and $22 \pm 1.1 \mu \mathrm{m}$ for BL-NAA ${ }_{(75 / 25)}$. Consequently, the length of the bottom layer was determined as $22 \pm 1.1 \mu \mathrm{m}$ for $\operatorname{BL}-N A A_{(25 / 75)}, 15 \pm 0.75 \mu \mathrm{m}$ for BL-NAA $(50 / 50)$, and BL-NAA $(75 / 25), 10 \pm 0.5 \mu \mathrm{m}$. Table 2 summarized the nanopore dimensions for BL-NAAs. It is observed that the bottom layers have smaller pore diameters which caused funnel like bi-layered structures. The bottom layers of nanostructures, 
BL-NAA $_{(25 / 75)}$, BL-NAA $_{(50 / 50)}$, BL-NAA $_{(75 / 25)}$ all showed a $45 \mathrm{~nm}$ pore diameter. The results of the SEM characterization can be explained due to the fact that the top layer formation of the bilayered structures occurred during the second step of anodization and the second step of anodization is responsible for bottom layer fabrication. It is also understood that the pore-widening process is the main reason that differences arise in pore diameters between the top layer and bottom layer which forms a funnel like structure. This was clearly proved where the control NAA $(1)$ and control $\mathrm{NAA}_{(2)}$ platforms revealed a single layer of nanostructure when one step of anodization and pore-widening process was eliminated.

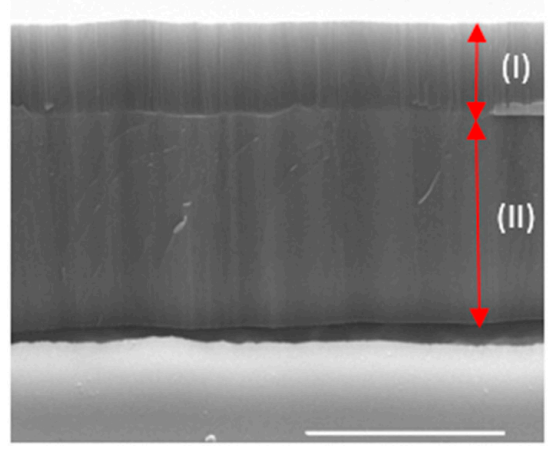

(a)

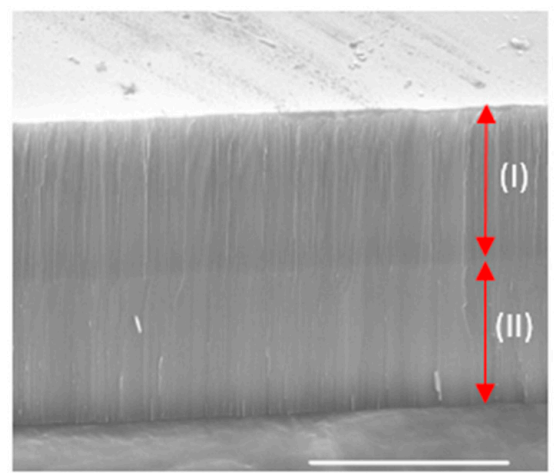

(c)

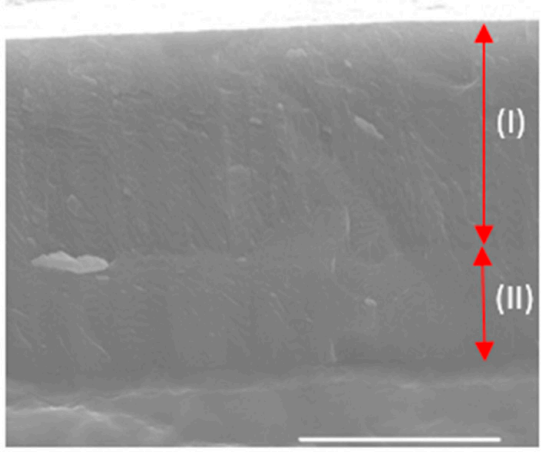

(e)

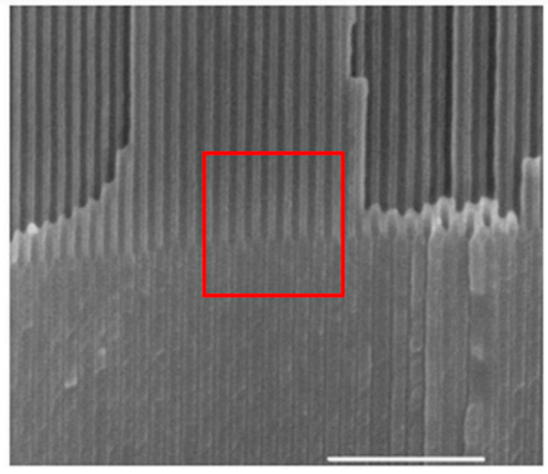

(b)

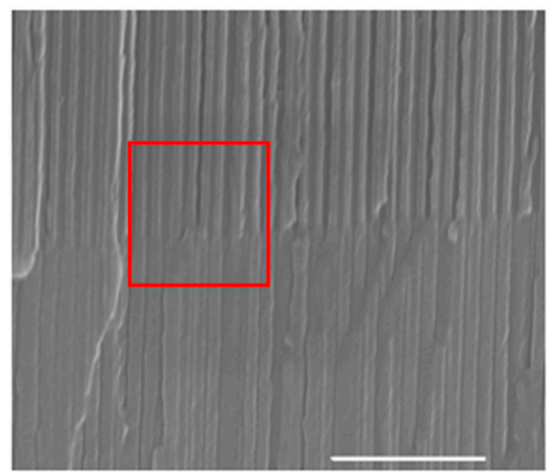

(d)

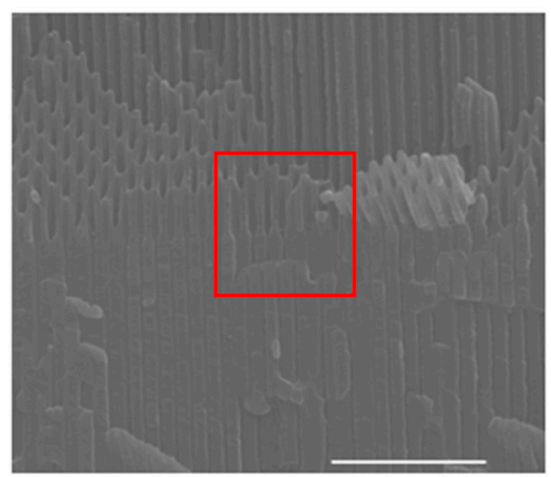

(f)

Figure 2. Scanning electron microscopy (SEM) structural characterization of fabricated BL-NAAs showing the length of pore layers and pore diameters. (a) Cross-section view of BL-NAA $(25 / 75)$, scale

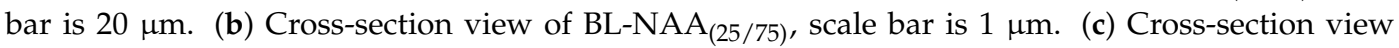
of BL-NAA $(50 / 50)$, scale bar is $20 \mu \mathrm{m}$. (d) Cross-section view of BL-NAA ${ }_{(50 / 50)}$, scale bar is $1 \mu \mathrm{m}$.

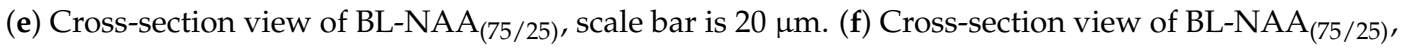
scale bar is $1 \mu \mathrm{m}$. 
Table 2. Pore dimensions of BL-NAAs and NAAs structures.

\begin{tabular}{|c|c|c|c|c|c|}
\hline \multicolumn{2}{|c|}{$\begin{array}{l}\text { Anodized Anodic Aluminum } \\
\text { Platforms }\end{array}$} & $\begin{array}{c}\text { Top Layer } \\
\text { Thickness }(\mu \mathrm{m})\end{array}$ & $\begin{array}{l}\text { Bottom Layer } \\
\text { Thickness }(\mu \mathrm{m})\end{array}$ & $\begin{array}{c}\text { Nanopore } \\
\text { thickness }(\mu \mathrm{m})\end{array}$ & $\begin{array}{c}\text { Pore Diameter } \\
\text { (Top/Bottom Layer) (nm) }\end{array}$ \\
\hline \multirow{3}{*}{ NAAF } & BL-NAA $_{(25 / 75)}$ & $9 \pm 0.45$ & $22 \pm 1.1$ & $30 \pm 1.5$ & $55 \pm 2.75 / 45 \pm 2.25$ \\
\hline & BL-NAA $_{(50 / 50)}$ & $15 \pm 0.75$ & $15 \pm 0.75$ & $30 \pm 1.5$ & $55 \pm 2.75 / 45 \pm 2.25$ \\
\hline & BL-NAA $_{(75 / 25)}$ & $22 \pm 1.1$ & $10 \pm 0.5$ & $32 \pm 1.6$ & $55 \pm 2.75 / 45 \pm 2.25$ \\
\hline \multirow{2}{*}{ NAA } & $\mathrm{NAA}_{(1)}$ & $\mathrm{N} / \mathrm{A}^{1}$ & $\mathrm{~N} / \mathrm{A}^{1}$ & $22 \pm 1.1$ & $55 \pm 2.75$ \\
\hline & $\mathrm{NAA}_{(2)}$ & $\mathrm{N} / \mathrm{A}^{1}$ & $\mathrm{~N} / \mathrm{A}^{1}$ & $23.5 \pm 1.17$ & $45 \pm 2.25$ \\
\hline
\end{tabular}

\subsection{Characterization and Optimization of RIfS Signals from BL-NAAs and NAAs Platforms}

As confirmed by SEM images, BL-NAA platforms feature a bilayered structure with larger pore diameters from the top, $55 \mathrm{~nm}$, to smaller pore diameter at the bottom, $45 \mathrm{~nm}$, with three different thicknesses of top and bottom layers. The fast Fourier transform (FFT) spectra of BL-NAAs, generated from optical interference pattern (see details of optical interference pattern in Supplementary Information), demonstrated more complex patterns than NAAs with a single layer structure. Figure 3 summarizes the FFT spectra of five different NAA structures analyzed in this study, including BL-NAA $_{(25 / 75)}$, BL-NAA $_{(50 / 50)}$, BL-NAA $_{(75 / 25)}, \mathrm{NAA}_{(1)}$, and NAA $\mathrm{N}_{(2)}$. FFT spectra from BL-NAA $_{(25 / 75)}$, BL-NAA $_{(50 / 50)}$, BL-NAA $_{(75 / 25)}$ (Figure $\left.3 a-c\right)$ feature three characteristic peaks with specific EOT with diverse FFT intensities that are used for sensing in this work. As can be seen, three peaks for BL-NAA $(25 / 75)$ are observed at $\mathrm{EOT}_{(\mathrm{I})}=38,000 \pm 1900 \mathrm{~nm}$, $\mathrm{EOT}_{(\mathrm{II})}=59,000 \pm 2950 \mathrm{~nm}$, and $\mathrm{EOT}_{(\mathrm{III})}=95,000 \pm 4750 \mathrm{~nm},{\mathrm{BL}-N A A_{(50 / 50)}}$ showed $\mathrm{EOT}_{(\mathrm{I})}=59,000 \pm 2950 \mathrm{~nm}, \mathrm{EOT}_{(\mathrm{II})}=61,000 \pm 3050 \mathrm{~nm}$, and $\mathrm{EOT}_{(\mathrm{III})}=120,000 \pm 6000 \mathrm{~nm}$, BL-NAA $_{(75 / 25)}$ also showed EOT $(\mathrm{I})=38,000 \pm 1900 \mathrm{~nm}, \mathrm{EOT}_{(\mathrm{II})}=71,000 \pm 3550 \mathrm{~nm}$, and $\mathrm{EOT}_{(\mathrm{III})}=107,000 \pm 5350 \mathrm{~nm}$. FFT peaks for $\mathrm{EOT}_{(\mathrm{I})}, \mathrm{EOT}_{(\mathrm{II})}$, and $\mathrm{EOT}_{(\mathrm{III})}$ correspond to the top, bottom, and total layers within the structure of BL-NAA platforms with three levels of light reflection. This result is in agreement with previous studies showing similar optical responses [32]. This phenomenon can be readily used to achieve unique sensing capabilities associated with EOT changes of each of these three peaks. In contrast to BL-NAAs, $\mathrm{NAA}_{(1)}$ and $\mathrm{NAA}_{(2)}$ showed a single peak in their FFT spectra, which is associated with the EOT of the nanoporous film (Figure 3d,e). Detection of quercetin using HSA-modified BL-NAAs and NAAs through RIfS was used to demonstrate the sensitivity of these optical platforms across all three optical layers. In this process, the EOT change obtained by applying FFT was used as sensing parameter to monitor in real-time the binding events occurring in each of the sensing layers.

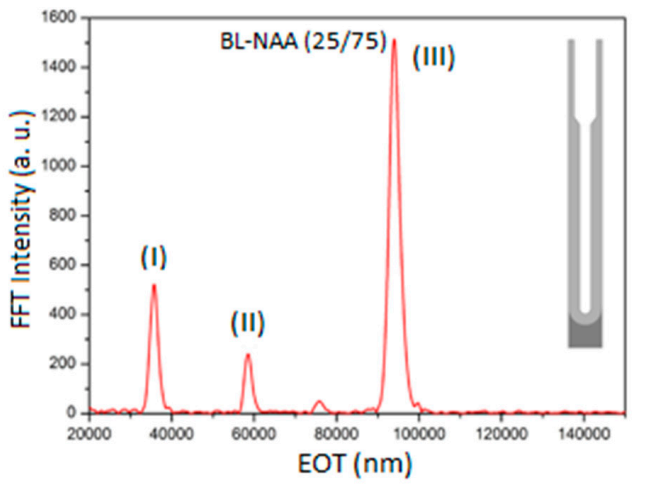

(a)

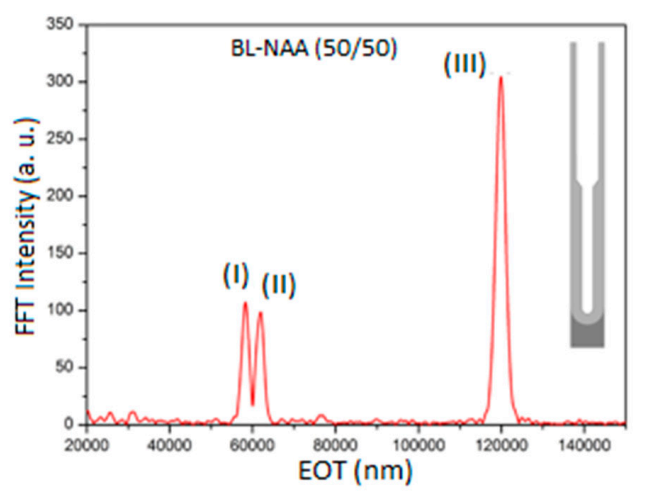

(b)

Figure 3. Cont. 


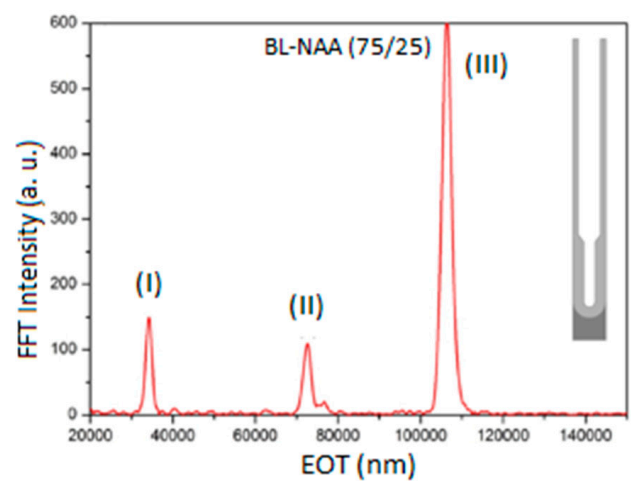

(c)

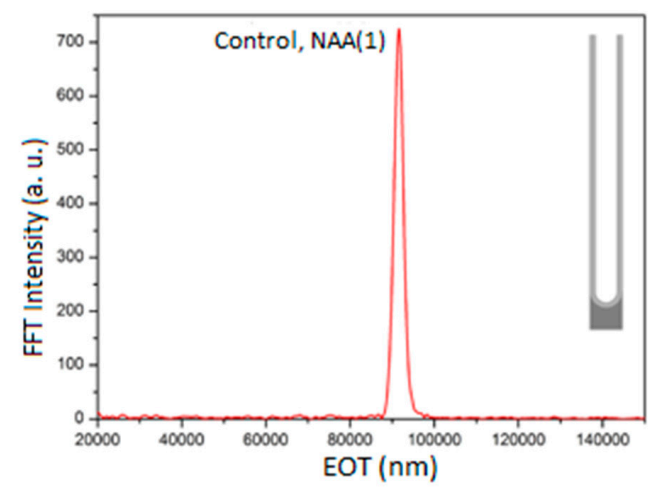

(d)

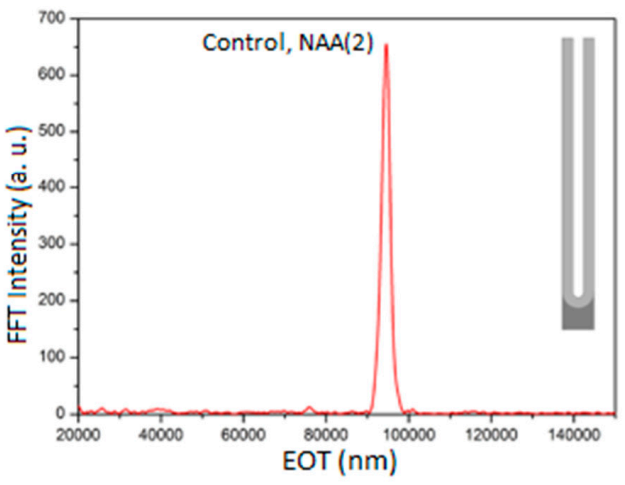

(e)

Figure 3. Comparison of FFT spectra of BL-NAAFs and NAAs with single layer and bilayer of nanoporous alumina structure. (a) Bi-layer structured of BL-NAA $(25 / 75)$ produced during (Step1 = $20 \mathrm{~h}$, step $2=3 \mathrm{~h}, 15 \mathrm{~min}$ pore widening, and step $3=6 \mathrm{~h}$ with no further pore widening), resulted in three optical peaks including (I), (II), and (II) various light reflection properties (e.g., FFT intensity and effective optical thickness); (b) Bi-layer structured of BL-NAA $(50 / 50)$ produced during (Step $1=20 \mathrm{~h}$, step $2=4.5 \mathrm{~h}$, $15 \mathrm{~min}$ pore widening, and step $3=4.5 \mathrm{~h}$ with no further pore widening), resulted in three optical peaks including (I), (II), and (II) various light reflection properties (e.g., FFT intensity and effective optical thickness); (c) Bi-layer structured of BL-NAA $_{(75 / 25)}$ produced during (Step $1=20 \mathrm{~h}$, step $2=6 \mathrm{~h}, 15 \mathrm{~min}$ pore widening, and step $3=3 \mathrm{~h}$ with no further pore widening), resulted in three optical peaks including (I), (II), and (II) various light reflection properties (e.g., FFT intensity and effective optical thickness). (d,e) Single-layer of control NAA $(1)$, and control NAA $(2)$ with a unique FFT.

\subsection{The Evaluation of Bl-NAA and NAA Sensing Platforms for Biomolecules Sensing}

Figure 4 illustrates the proposed molecular distribution and binding reaction of sensing molecules inside larger and smaller pores of BL-NAA and continuous RIfS signals ( $\triangle \mathrm{EOT}$ as the sensing parameter) obtained during different stages of our study to assess the sensing performance of BL-NAA platforms. This sensing process is based on real-time monitoring of EOT of each corresponding nanoporous layers that were monitored through all steps from the fabrication of sensing surface to final sensing of analyte molecules (Figure $4 \mathrm{~b}$ ). The following steps are observed in the figure including (i) amine (-NH 2$)$ group activation by GTA; (ii) HSA functionalization of inner surface of BL-NAAs and NAAs; and (iii) quercetin binding reaction to HSA. Changes of EOT were observed during GTA, HSA and quercetin flow into the system which means the binding reaction between the molecules and the inner surface of BL-NAAs. Finally, sensing of quercetin molecules revealed stable and higher EOT where all HSA molecules are saturated and no further binding reaction occurred showing the sensing capabilities of this system. 


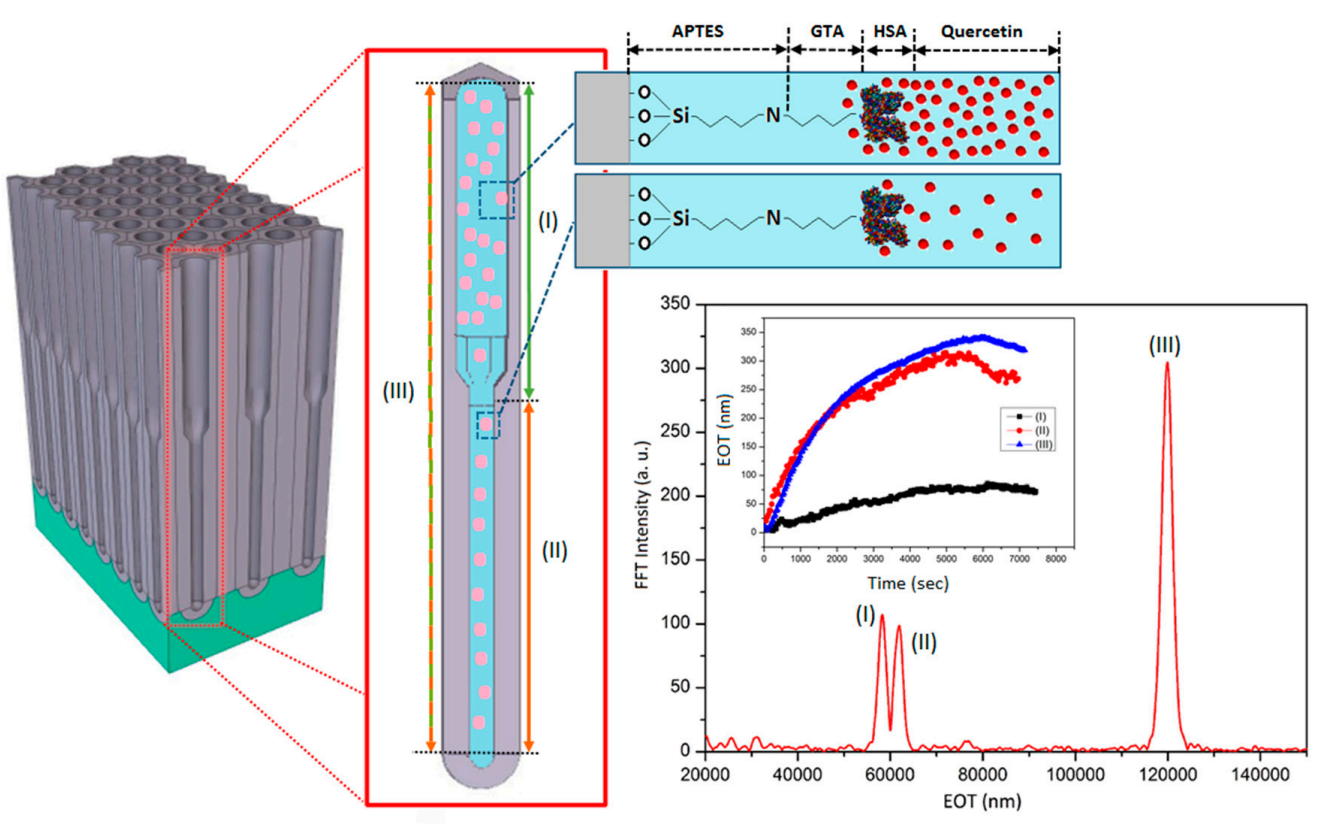

(a)

(b)

Figure 4. The proposed RIfS sensing concept using BL-NAAs platforms showing changes of EOT signals during surface modification and the preparation of sensing layer (HSA) and sensing analyte molecules (quercetin) showing the binding activity between HSA-modified pore surface and quercetin. (a) Schematic illustration of binding event between HSA and quercetin in the environment of fresh PBS $(\mathrm{pH}=7.5)$ in the top and bottom pore layer. BL-NAAs and NAAs were modified with silane molecules before subjecting them to real-time monitoring process; (b) typical FFT spectra of BL-NAA $(50 / 50)$ showing 3 distinguished peaks that are related to two pore layers and used for sensing showing time response RIfS signal that present EOT measurement quercetin $(0.375 \mathrm{mM})$ binding activity in all 3 optical layers simultaneously.

The obtained results for $\triangle \mathrm{EOT}$ for each analytical solution of quercetin $(0.05,0.125,0.25,0.375$, 0.5 , and $1 \mathrm{mM}$ ) using BL-NAAs with different layer thicknesses and control NAAs are displayed and summarized in Figures 5-8 and Table 3 . The calculation of linear fitting lines between $\triangle \mathrm{EOT}$ and the concentration of quercetin was used to estimate the sensitivity, $\mathrm{S}$ which is the slope of the fitting lines, the low limit of detection, LLOD which is calculated as $3 \sigma$ according the Equation (2), and the linearity, $R^{2}$ which is the correlation coefficient of the fitting line, for each BL-NAA and NAA (Table 3).

$$
\text { LLOD }=3 \sigma=3.3 \text { standard error } \div \text { slope }
$$

Figure 5 presents the $\Delta$ EOTs of BL-NAA $(25 / 75)$ platform with a funnel-like structure consisting of a shorter top layer of large pore diameter (Figure $5 \mathrm{a}$ ) anda longer bottom layer featuring a smaller pore diameter obtained from the top layer I and combined top and bottom layer (Figure 5d). Generally, the $\Delta \mathrm{EOT}_{\text {(III) }}$ signal from combined top and bottom layer (optical layer 3) showed higher values compared to $\Delta \mathrm{EOT}_{(\mathrm{I})}$ taken from the first layers. The $\Delta \mathrm{EOT}_{(\mathrm{I})}$ and $\Delta \mathrm{EOT}_{(\mathrm{III})}$ were calculated with maximum changes of $300 \mathrm{~nm}$ and $450 \mathrm{~nm}$, respectively and were obtained for the highest concentration of quercetin $1 \mathrm{mM}$ (Figure $5 \mathrm{a}, \mathrm{d}$ ). Notice that the $\triangle \mathrm{EOT}_{(\mathrm{II})}$ measurement from the bottom layer is not included during investigation of this type of BL-NAA platform due to the lack of sufficient optical efficiency of the bottom layer of the $\mathrm{BL}-\mathrm{NAA}_{(25 / 75)}$ platform which caused failure in the EOT screening. Calibration graphs also showed that the $\triangle E O T$ has a linear dependence with quercetin concentrations (Figure 5b,e). Comparative calibration graphs (Figure $5 b$ vs. 5e) also showed a higher precision of measurement taken from optical layer III evidenced by the correlation factors $R^{2}$ BL-NAA $_{(25 / 75)}(\mathrm{I})=0.922$ and BL-NAA $(25 / 75)($ III $)=0.948$ (Table 3). It is seen that layer (III), has a 
smaller dispersion around the fitting line compared to layer (I), indicating a stronger linear relationship of layer (III) than layer (I) (Figure $5 \mathrm{~b}, \mathrm{e})$. The sensing performance evaluation of BL-NAA $(25 / 75)$ resulted in values of $S_{(\mathrm{I})}=378 \pm 48\left(\mathrm{~nm} \mathrm{mM}^{-1}\right)$ and $S_{(\mathrm{III})}=418 \pm 43\left(\mathrm{~nm} \mathrm{mM}^{-1}\right)$ for BL-NAA $(25 / 75)$ (I) and

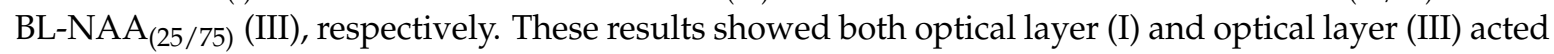
as highly sensitive sensing areas with the ability of $L L O D_{(\mathrm{I})}=0.034 \mathrm{mM}$ and $L L O D_{(\mathrm{III})}=0.074 \mathrm{mM}$ quercetin detection.

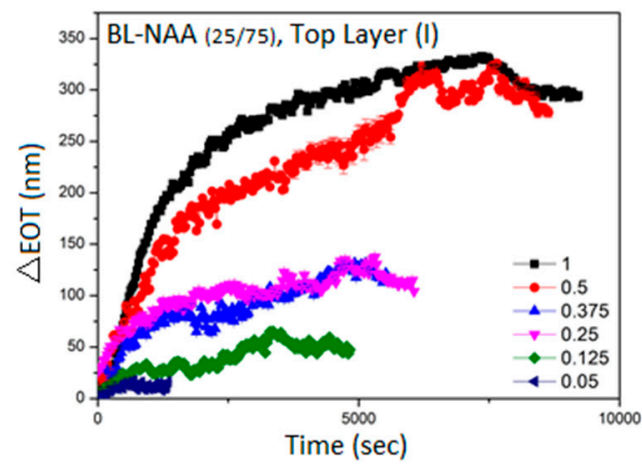

(a)

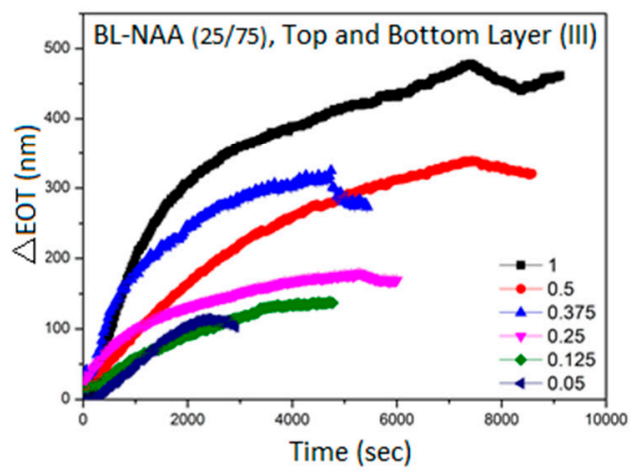

(d)

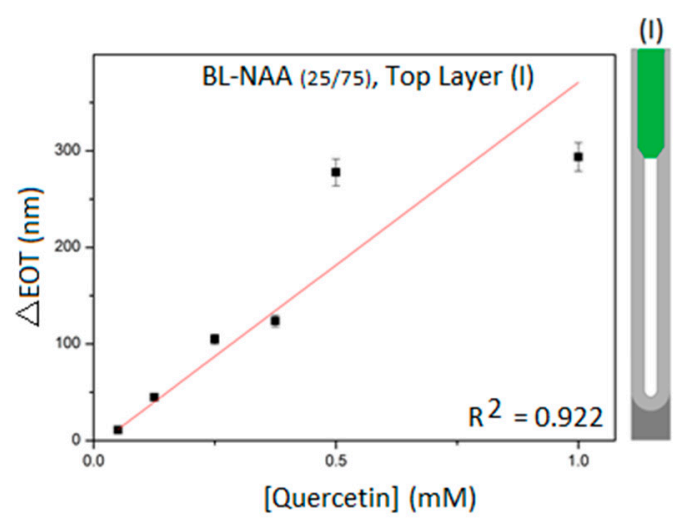

(b)

(c)

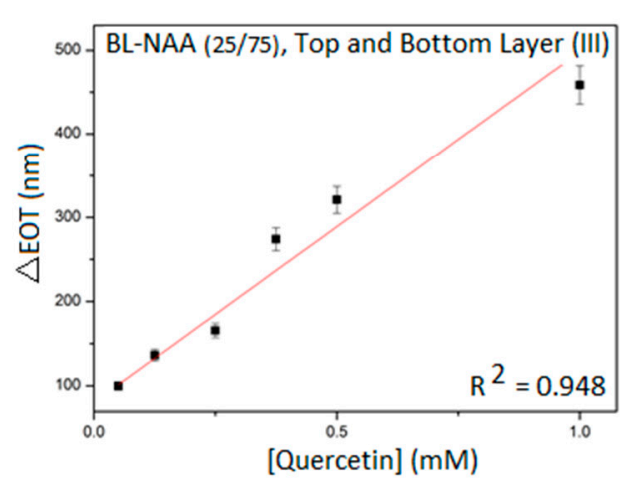

(e)

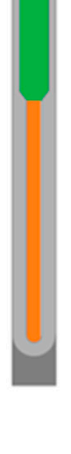

(f)

Figure 5. Time response curves showing effective optical thickness (EOT) changes and Linear fitting line for BL-NAA $(25 / 75)$ (funnel structure with shorter top layer with large pore diameters and longer bottom layer with smaller pore diameters) sensing platforms measured by RIfS as a function of the quercetin concentration $(0.05,0.125,0.25,0.375,0.5$, and $1 \mathrm{mM})$. (a) $\Delta \mathrm{EOT}_{\text {(I) }}$ generated from the

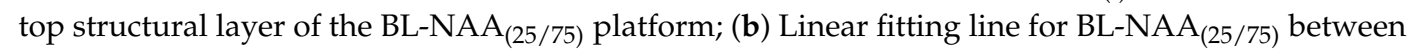
$\Delta \mathrm{EOT}_{(\mathrm{I})}$ and the different concentrations of quercetin $(0.05,0.125,0.25,0.375,0.5$, and $1 \mathrm{mM})$; (c) Scheme of the representative optical layer as a function of $\Delta \mathrm{EOT}_{(\mathrm{I})}$ assessment; (d) $\Delta \mathrm{EOT}_{(\mathrm{III})}$ generated from

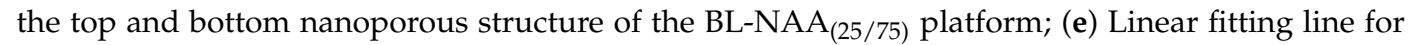
BL-NAA $_{(25 / 75)}$ between $\Delta \mathrm{EOT}_{(\mathrm{III})}$ and the different concentration of quercetin $(0.05,0.125,0.25,0.375$, 0.5 , and $1 \mathrm{mM}$ ). (f) Scheme of representative optical layer as a function of $\Delta \mathrm{EOT}_{(\mathrm{III})}$ assessment.

Figure 6 summarizes the response time graphs of $\triangle \mathrm{EOTs}$ of $\mathrm{BL}-\mathrm{NAA}_{(50 / 50)}$, platform (funnel structure equal length of top layer of large diameters and longer layer with smaller pore diameters) obtained from layer (I), layer (II), and layer (III) using a series of standard quercetin concentrations. These results were used to make corresponding calibration curves with schematic illustrations of a singular nanopore structure highlighting layer (I) in green color, layer (II) in orange color and layer (III) in green/orange color used for sensing in each graph. The time response graphs, for each optical layer (The $\Delta \mathrm{EOT}_{(\mathrm{I})}, \Delta \mathrm{EOT}_{(\mathrm{II})}$ and $\Delta \mathrm{EOT}_{(\mathrm{III})}$ ) showed different values for the maximum concentration of quercetin ( $1 \mathrm{mM}$ ) starting from $120 \mathrm{~nm}, 270 \mathrm{~nm}$, and $420 \mathrm{~nm}$, respectively, (Figure 6a,d,g) indicating 
a different sensitivity for each layer. The sigmoidal curve fitting shows that the $\Delta \mathrm{EOT}$ has a sigmoidal dependence with the concentration of quercetin (Figure $6 \mathrm{~b}, \mathrm{e}, \mathrm{h}$ ). However, there are linear correlations amongst quercetin concentrations (i.e., $0.25,0.375$, and $0.5 \mathrm{mM}$ ). The dispersion of those data points represents similar $R^{2}$ values of the top layer (I), bottom layer (II) and top and bottom layer (III) which

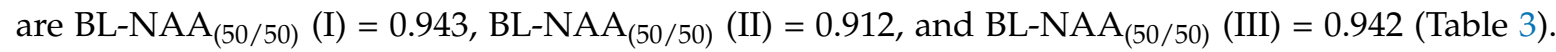
The sensing performance of $\mathrm{BL}_{-\mathrm{NAA}}(50 / 50)$, evaluated by sensitivity graphs, showed values of $S_{(\mathrm{I})}=389 \pm 66\left(\mathrm{~nm} \mathrm{mM}^{-1}\right), S_{(\mathrm{II})}=462 \pm 70\left(\mathrm{~nm} \mathrm{mM}^{-1}\right)$, and $S_{(\mathrm{III})}=1207 \pm 208 S\left(\mathrm{~nm} \mathrm{mM}^{-1}\right)$ for

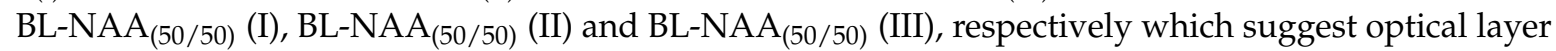
(III) acted as a highly sensitive sensing area, and optical layer (I) and (II) did not act as high sensitive sensing areas. Optical layer (I) was the least sensitive when compared to two other optical layers. $L L O D_{(\mathrm{I})}=0.148 \mathrm{mM}, L L O D_{(\mathrm{II})}=0.1 \mathrm{mM}$, and $L L O D_{(\mathrm{III})}=0.168 \mathrm{mM}$ were evaluated for the limit of quercetin detection. The sensitivity of optical layer III compared with results of the funnel with a shorter top layer significantly decreased from $S_{(\mathrm{III})}=1207 \pm 208$ to $S_{(\mathrm{III})}=418 \pm 43 \mathrm{~S}\left(\mathrm{~nm} \mathrm{mM}^{-1}\right)$.

Figure 7 summarizes response time graphs of $\triangle$ EOTs based on BL-NAA $(75 / 25)$, platform (funnel structure with longer thickness of top layer of large diameters and shorter layer with smaller pore diameters) obtained from layer (I), layer (II), and layer (III) using BL-NAA (50/50), and a series of standard quercetin concentrations. The pertinent calibration curves are shown of $\triangle \mathrm{EOT}$ and quercetin concentrations, with schematic illustrations of the singular nanopore structure highlighting layer (I) in green color, layer (II) in orange color and layer (III) in green/orange color. According to the results,

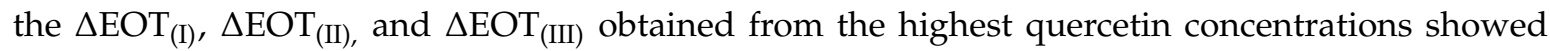
maximum changes of $100 \mathrm{~nm}, 370 \mathrm{~nm}$, and $460 \mathrm{~nm}$, respectively (Figure 7a,d,g) which suggests the highest sensitivity for optical layer III. The calibration graph shows that $\triangle \mathrm{EOT}$ has linear dependence with the all concentration of quercetin of layer (I) (Figure $7 \mathrm{~b}$ ). However, there is a sigmoid correlation with the concentrations of the quercetin of layer (II) and layer (III) (Figure 7e,h). The dispersion of data points around the fitting curves for $\Delta \mathrm{EOT}_{(\mathrm{I})} \Delta \mathrm{EOT}_{(\mathrm{II})}$ represents a strong correlation between the top layer (I) and bottom layer (II). Evidenced by the calculated $R^{2}$ are BL-NAA ${ }_{(75 / 25)}(\mathrm{I})=0.841$, BL-NAA $_{(75 / 25)}(\mathrm{II})=0.857$, and BL-NAA $(75 / 25)(\mathrm{III})=0.793$, presented in Table 3.

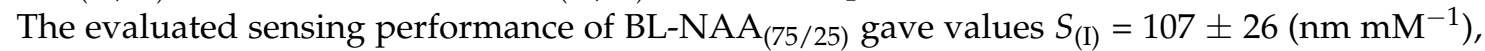
$S_{(\mathrm{II})}=884 \pm 245\left(\mathrm{~nm} \mathrm{mM}^{-1}\right)$ and $S_{(\mathrm{III})}=670 \pm 227 S\left(\mathrm{~nm} \mathrm{mM}^{-1}\right)$ for BL-NAA $(75 / 25)(\mathrm{I}), \mathrm{BL}-\mathrm{NAA}_{(75 / 25)}$ (II) and BL-NAA $(75 / 25)$ (III), respectively. These results showed that optical layers (III) and (II) acted as highly sensitive sensing areas, and that optical layer (I) did not act as a high sensitive sensing area. $L L O D_{(\mathrm{I})}=0.179 \mathrm{mM}, L L O D_{(\mathrm{II})}=0.160 \mathrm{mM}$ and $L L O D_{(\mathrm{III})}=0.256 \mathrm{mM}$ are evaluated for the limit of quercetin detection.

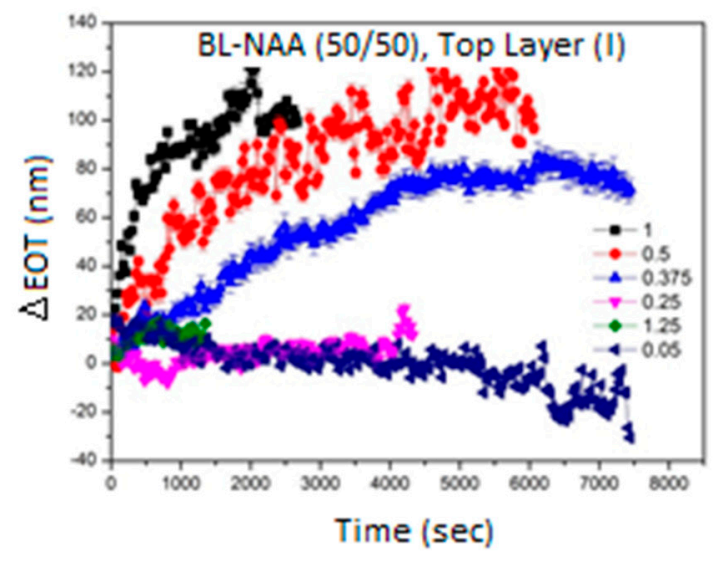

(a)

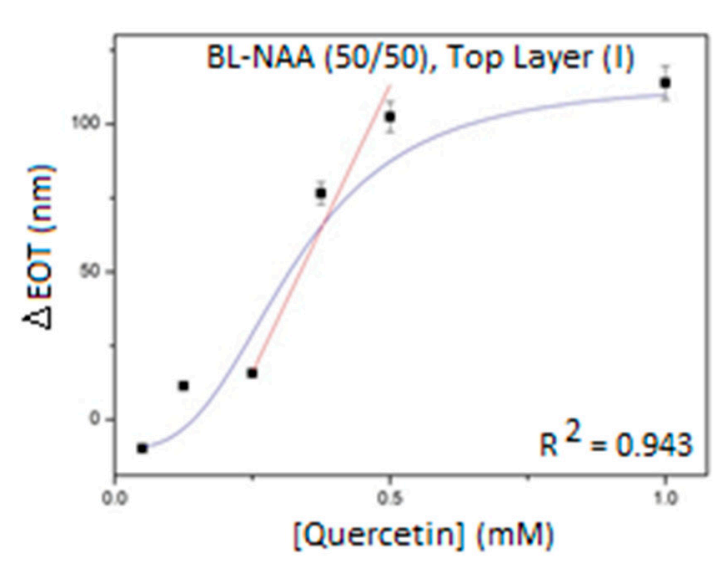

(b)
(I)

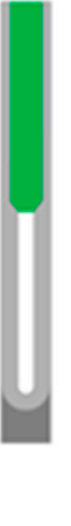

(c)

Figure 6. Cont. 


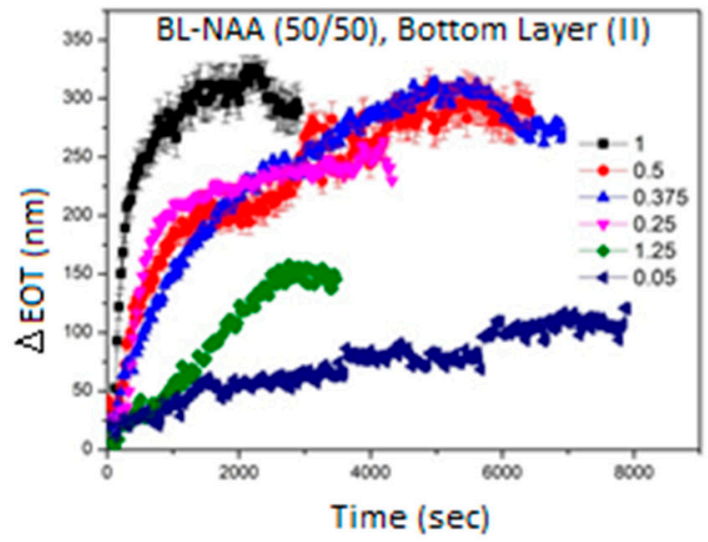

(d)

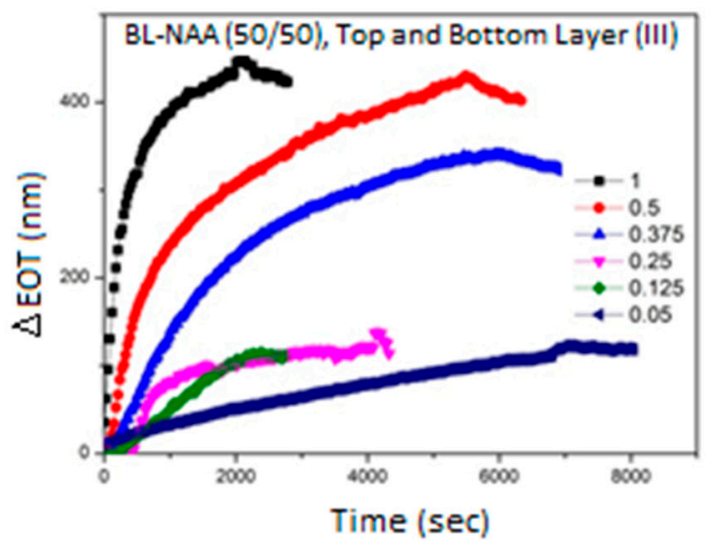

(g)

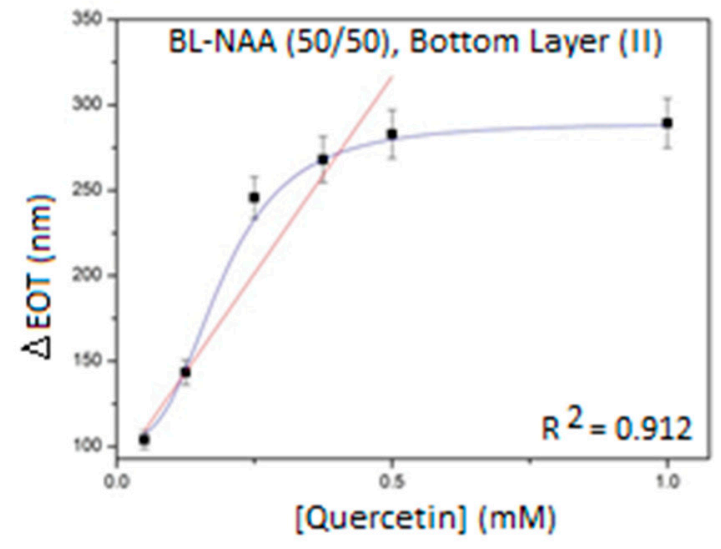

(e)

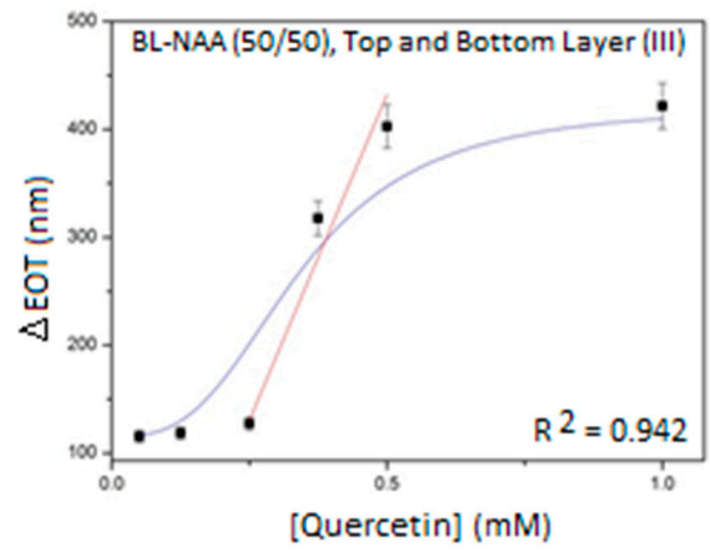

(h)
(II)

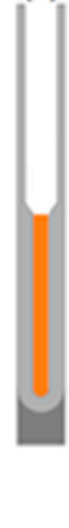

(f)

(III)

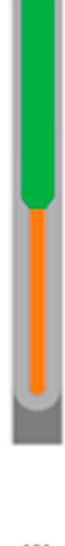

(i)

Figure 6. Time response curve showing effective optical thickness changes and sigmoidal curve

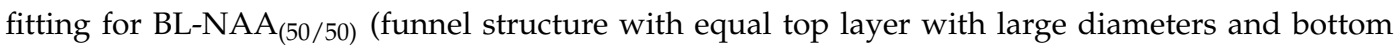
layer with smaller pore diameters) sensing platforms measured by RIfS as a function of quercetin concentration $(0.05,0.125,0.25,0.375,0.5$, and $1 \mathrm{mM})$. (a) $\Delta \mathrm{EOT}_{(\mathrm{I})}$ generated from the top structural layer of the BL-NAA $(50 / 50)$ platform; (b) Sigmoid fit (blue) and the linear fit (red) for BL-NAA $(50 / 50)$ between $\triangle \mathrm{EOT}_{(\mathrm{I})}$ and the different concentration of quercetin $(0.05,0.125,0.25,0.375,0.5$, and $1 \mathrm{mM})$ are presented; (c) Scheme of representative optical layer as a function of $\Delta \mathrm{EOT}_{(\mathrm{I})}$ assessment; (d) $\Delta \mathrm{EOT}_{\text {(II) }}$

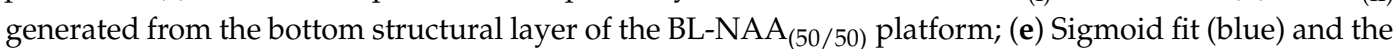
linear fit (red) for BL-NAA $(50 / 50)$ between $\Delta \mathrm{EOT}_{\text {(II) }}$ and the different concentration of quercetin $(0.05$, $0.125,0.25,0.375,0.5$, and $1 \mathrm{mM}$ ) are presented; (f) Scheme of representative optical layer as a function of $\Delta \mathrm{EOT}_{(\mathrm{II})}$ assessment; (g) $\Delta \mathrm{EOT}_{(\mathrm{III})}$ generated from the top and bottom nanoporous structure of BL-NAA $_{(50 / 50)}$ platform; (h) Sigmoid fit (blue) and the linear section (red) for BL-NAA $(50 / 50)$ between $\Delta \mathrm{EOT}_{\text {(III) }}$ and the different concentration of quercetin $(0.05,0.125,0.25,0.375,0.5$, and $1 \mathrm{mM})$ are presented; (i) Scheme of representative optical layer as a function of $\Delta \mathrm{EOT}_{(\mathrm{III})}$ assessment.

Figure 8 summarizes response time $\triangle \mathrm{EOTs}$ graphs based on the single layer of $\mathrm{NAA}_{(1)}$ and $\mathrm{NAA}_{(2)}$, used as control corresponding to calibration graphs and schematic illustrations of the singular nanopore structure highlighted in brown color. According to the results, $\triangle E O T$ of NAA $(1)$ shows higher values compared to $\triangle \mathrm{EOT}$ of $\mathrm{NAA}_{(2)}($ Figure $8 \mathrm{a}, \mathrm{d})$. These graphs also show that $\triangle \mathrm{EOT}$ have a sigmoid dependence with the concentration of quercetin (Figure $8 \mathrm{~b}, \mathrm{e}$ ). The dispersion of data points around the curve for the $\triangle \mathrm{EOT}$ of $\mathrm{NAA}_{(1)}$ represents a stronger correlation compared with the $\triangle \mathrm{EOT}$ for $\mathrm{NAA}_{(2)}$, evidenced by $\mathrm{R}^{2} 0.946$ for $\mathrm{NAA}_{(1)}$ and 0.799 for $\mathrm{NAA}_{(2)}$ (Table 3). The sensing performance evaluation of $\mathrm{NAA}_{(1)}$ resulted in a value of $S=842 \pm 139\left(\mathrm{~nm} \mathrm{mM}^{-1}\right)$. These results showed that 
the single optical layer of $\mathrm{NAA}_{(1)}$ acted as a highly sensitive sensing platform. $L L O D=0.188 \mathrm{mM}$ of $\mathrm{NAA}_{(1)}$ is evaluated for the limit of quercetin detection. The sensing performance evaluation of $\mathrm{NAA}_{(2)}$ resulted in a value of $S=521 \pm 174\left(\mathrm{~nm} \mathrm{mM}^{-1}\right)$. These results showed the single optical layer of $\mathrm{NAA}_{(2)}$ did not act as a highly sensitive sensing platform. The $L L O D=0.449 \mathrm{mM}$ of NAA(2) was evaluated for the limit of quercetin detection. Control NAA $(1)$ showed a higher level of sensitivity which means a larger pore diameter can affect the sensing performance.

The obtained sensitivity results of the overall optical sensing performance for BL-NAAs and NAAs in this study are summarized in Figure 9 and Table 3. According to the data, optical layer (I) of BL-NAA $_{(25 / 75)}$ showed almost similar $S$ with the optical layer (I) of BL-NAA $(50 / 50)$ whilst optical layer (I) of BL-NAA $(75 / 25)$ showed a lower $S$ than optical layer (I) of BL-NAA $(50 / 50)$ and

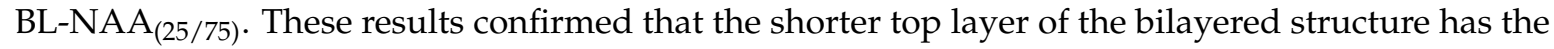
better sensitivity and stronger optical capability. The optical layer (II) of BL-NAA $(75 / 25)$ revealed the highest sensitivity compared to optical layers (II) of BL-NAA $(25 / 75)$ and BL-NAA $(50 / 50)$ which further leads us to the conclusion that the shorter structural layer in a bilayered structure caused higher sensitivity. This is an important conclusion that should be considered when designing optimal sensing of multilayered NAA structures. Therefore, thick and in-depth fabrication of bilayered nanostructures reduced the optical characteristics of nanostructures, as seen by failed optical screening for optical layer (II) of BL-NAA $(25 / 75)$. Interestingly, optical layer (III) of BL-NAA $(50 / 50)$ showed highest sensitivity compared to optical layers (III) of BL-NAA $(25 / 75)$, BL-NAA $_{(75 / 25)}$. The value of sensitivities for optical layer (III) of BL-NAA $(50 / 50)$ was higher compared to the single layer structures used in this study $\left(\mathrm{NAA}_{(1)}\right.$ and $\left.\mathrm{NAA}_{(2)}\right)$. Therefore, $\mathrm{BL}^{-\mathrm{NAA}}(50 / 50)$ can be the proper choice of optimized bilayered structures due to its capability in multi-point sensing with high sensitivity at each layer. Additionally, control $\mathrm{NAA}_{(1)}$ depicted a single path of $\triangle \mathrm{EOT}$ screening. Control NAAs demonstrated the significant role of pore widening in sensing performance, where a lack of pore widening process diminishes $S$, significantly. Considerably, BL-NAAs could establish the multiple sensing pathways of a diverse range of sensing features. The sensitivities of these five nanoporous anodic alumina platforms are summarized in Figure 9.

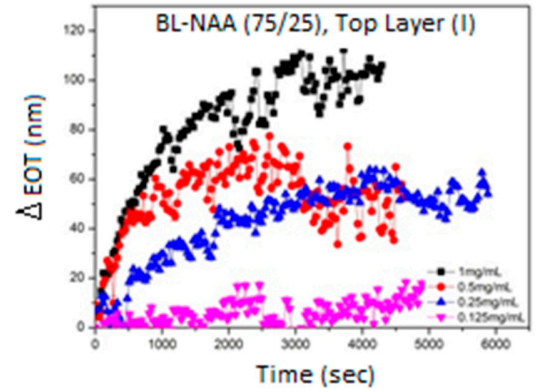

(a)

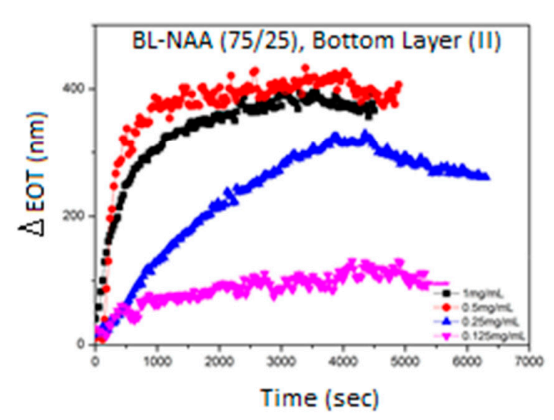

(d)

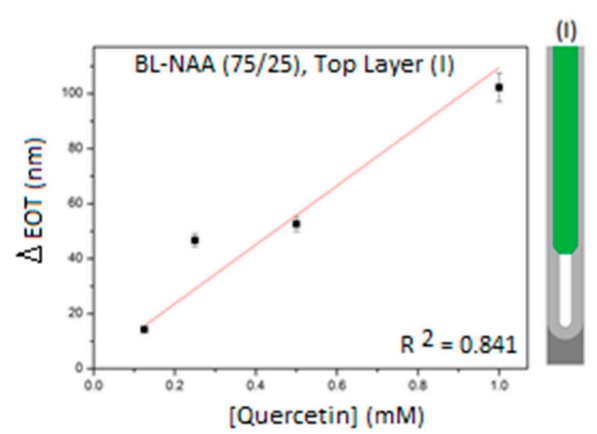

(b)

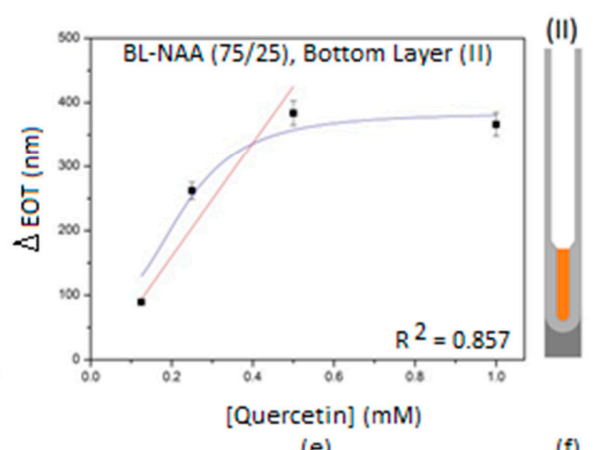

(e) (c)

(II)

Figure 7. Cont. 


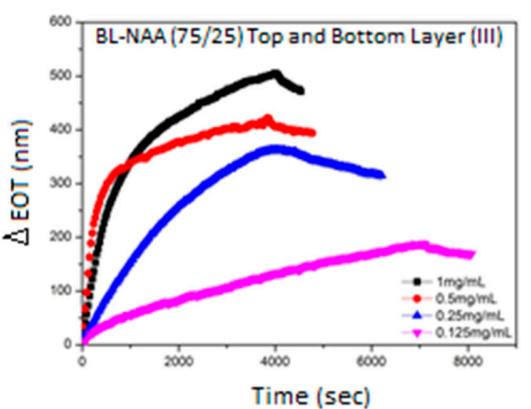

(g)

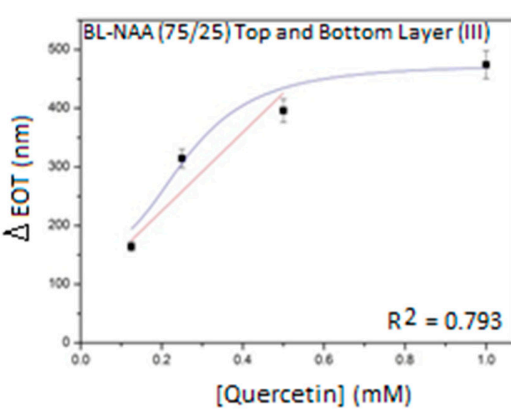

(h)
(III)

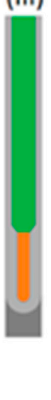

(i)

Figure 7. Time response curve showing effective optical thickness changes and fitting curves for BL-NAA $_{(75 / 25)}$ (funnel structure with longer top layer with large diameters and shorter bottom layer with smaller pore diameters) sensing platforms measured by RIfS as a function of quercetin concentration $(0.125,0.25,0.5$, and $1 \mathrm{mM})$. (a) $\Delta \mathrm{EOT}_{(\mathrm{I})}$ generated from top structural layer of BL-NAA $_{(75 / 25)}$ platform; (b) Linear fitting line for BL-NAA B $_{(75 / 25)}$ between $\Delta \mathrm{EOT}_{(\mathrm{I})}$ and the different concentration of quercetin $(0.125,0.25,0.5$, and $1 \mathrm{mM})$; (c) Scheme of representative optical layer as a function of $\Delta \mathrm{EOT}_{(\mathrm{I})}$ assessment; (d) $\Delta \mathrm{EOT}_{(\mathrm{II})}$ generated from bottom structural layer of BL-NAA $\mathrm{A}_{(75 / 25)}$ platform; (e) Sigmoid fit (blue) and the linear fit (red) for BL-NAA B $_{(55 / 25)}$ between $\triangle \mathrm{EOT}$ (II) and the different concentration of quercetin $(0.125,0.25,0.5$, and $1 \mathrm{mM})$ are presented; (f) Scheme of representative optical layer as a function of $\Delta \mathrm{EOT}_{(\mathrm{II})}$ assessment; (g) $\Delta \mathrm{EOT}_{(\mathrm{III})}$ generated from the top and bottom nanoporous structure of the BL-NAA $(75 / 25)$ platform; (h) Sigmoid fit (blue) and the linear fit (red) for BL-NAA ${ }_{(75 / 25)}$ between $\triangle E O T$ (III) and the different concentration of quercetin $(0.05,0.125$, $0.25,0.375,0.5$, and $1 \mathrm{mM}$ ) are presented; (i) Scheme of representative optical layer as a function of $\Delta \mathrm{EOT}_{(\mathrm{III})}$ assessment.

Table 3. Result of sensing performance of BL-NAAs and NAAs by $\triangle E O T$ measurement.

\begin{tabular}{|c|c|c|c|c|}
\hline Sample & Optical Layer & $S\left(\mathrm{~nm} \mathrm{~m}^{-1}\right)$ & $L L O D(\mathrm{mM})$ & $R^{2}$ \\
\hline \multirow{3}{*}{ BL-NAA $_{(25 / 75)}$} & (I) & $378 \pm 48$ & 0.034 & 0.922 \\
\hline & (II) & $\mathrm{ND}^{1}$ & $\mathrm{ND}^{1}$ & $\mathrm{ND}^{1}$ \\
\hline & (III) & $418 \pm 43$ & 0.074 & 0.948 \\
\hline \multirow{3}{*}{ BL-NAA $_{(50 / 50)}$} & (I) & $389 \pm 66$ & 0.148 & 0.943 \\
\hline & (II) & $462 \pm 70$ & 0.1 & 0.912 \\
\hline & (III) & $1207 \pm 208$ & 0.168 & 0.942 \\
\hline \multirow{3}{*}{ BL-NAA $_{(75 / 25)}$} & (I) & $107 \pm 26$ & 0.179 & 0.841 \\
\hline & (II) & $884 \pm 245$ & 0.160 & 0.857 \\
\hline & (III) & $670 \pm 227$ & 0.256 & 0.793 \\
\hline $\mathrm{NAA}_{(1)}$ & Single layer & $842 \pm 139$ & 0.188 & 0.946 \\
\hline $\mathrm{NAA}_{(2)}$ & Single layer & $521 \pm 174$ & 0.449 & 0.799 \\
\hline
\end{tabular}

${ }^{1}$ ND means Not Detectable. 


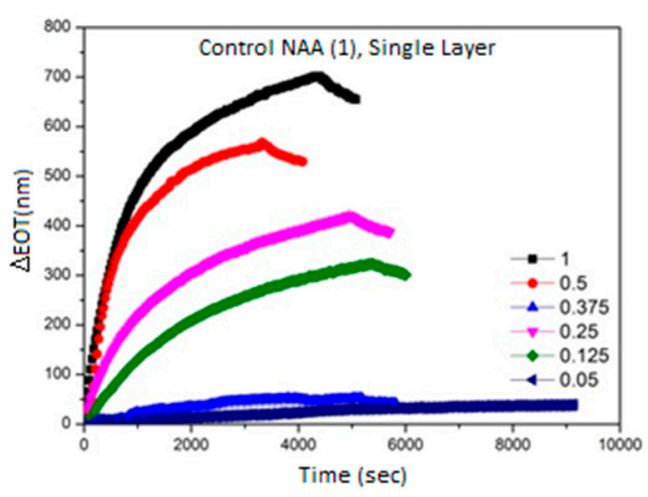

(a)

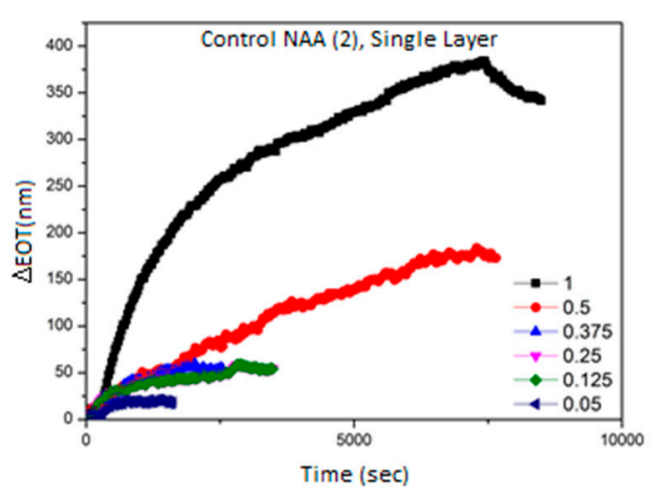

(d)

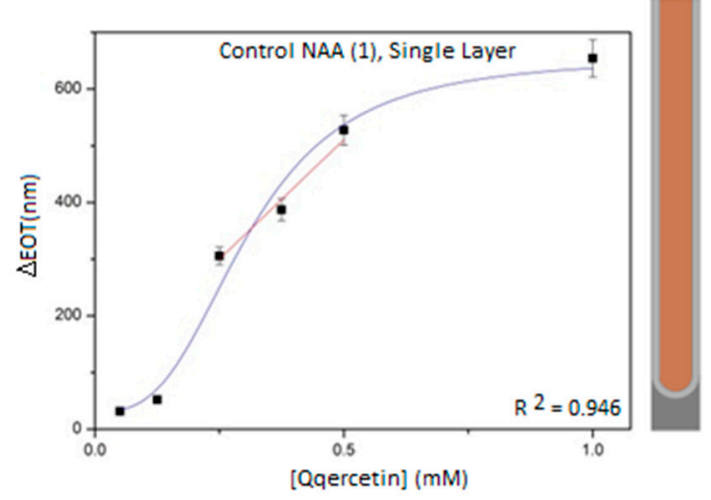

(b)

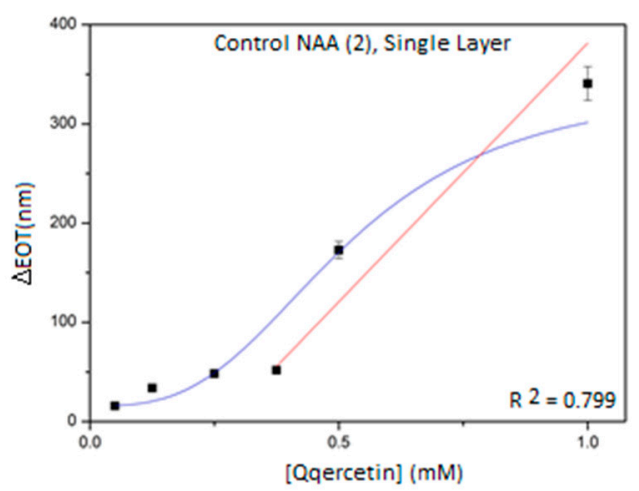

(e) (c)

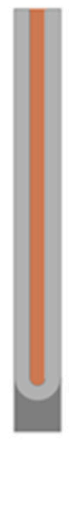

(f)

Figure 8. Time response curves showing effective optical thickness changes and fitting curves for control $\mathrm{NAA}_{(1)}$ and $\mathrm{NAA}_{(1)}$ sensing platforms measured by RIfS as a function of quercetin concentration $(0.05,0.125,0.25,0.375,0.5$, and $1 \mathrm{mM})$. (a) $\triangle \mathrm{EOT}$ generated from single layer nanoporous structure of $\mathrm{NAA}_{(1)}$ platform; (b) Sigmoid fit (blue) and the linear fit (red) for $\mathrm{NAA}_{(1)}$ between $\triangle \mathrm{EOT}$ and the different concentration of quercetin $(0.05,0.125,0.25,0.375,0.5$, and $1 \mathrm{mM})$ are presented; (c) Scheme of representative optical layer as a function of $\triangle \mathrm{EOT}$ assessment; (d) $\triangle \mathrm{EOT}$ generated from single layer nanoporous structure of $\mathrm{NAA}_{(2)}$ platform; (e) Sigmoid fit (blue) and the linear fit (red) for NAA(2) between $\triangle E O T$ and the different concentration of quercetin $(0.05,0.125,0.25,0.375,0.5$, and $1 \mathrm{mM})$ are presented; (f) Scheme of representative optical layer as a function of $\Delta \mathrm{EOT}$ assessment.

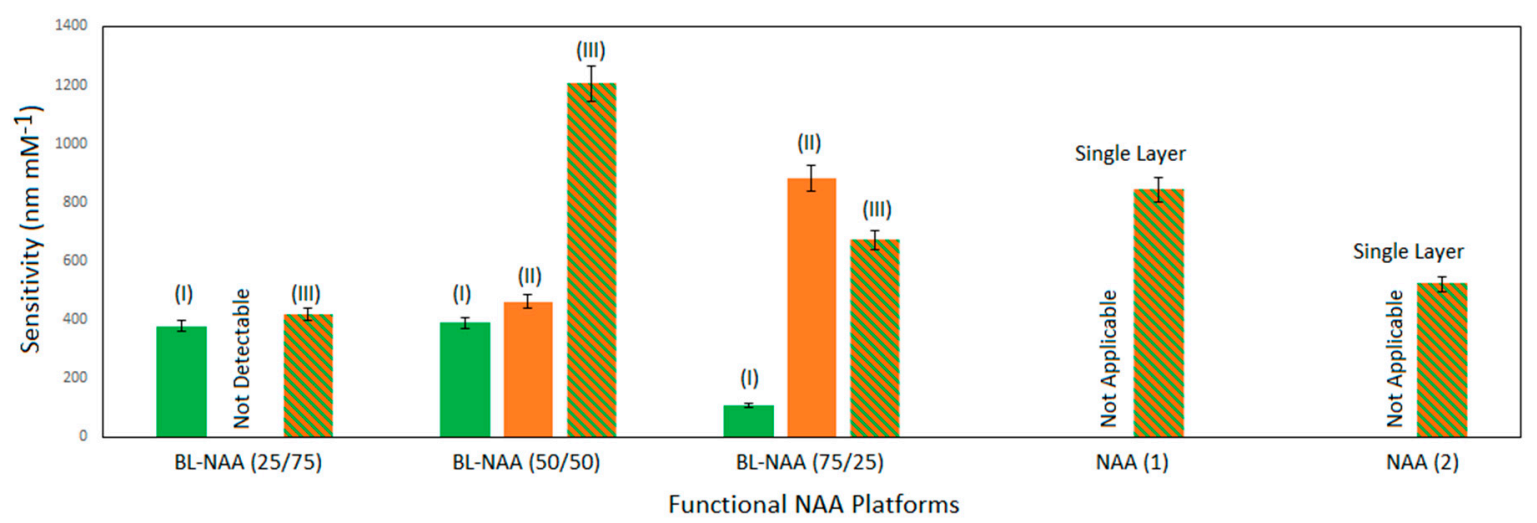

Figure 9. Bar chart summarizing the sensitivities (i.e., the slope of linear fittings shown in Figures 5-8) for BL-NAAs and NAAs sensing platforms modified with HSA-quercetin. 


\section{Conclusions}

In this work fabrication of bilayered NAA with a funnel-like nanopore structure (larger pore diameter on top and smaller pore diameter on the bottom) was successfully demonstrated using a combination of sequential anodization steps and pore widening process and was confirmed by systematic structural SEM characterizations. The bilayered nanostructures present complex RIfS and FFT spectra, which can be readily and independently used as a sensing pattern. A set of three BL-NAAs platforms with different lengths of porous layers were evaluated to establish the most sensitive pore structures for a quercetin molecule, a model plant flavonol, and detection as a function of three optical layers (i.e., optical layer I, optical layer II, optical layer III). The design of sensing strategy made it possible to establish the effect of each optical layer on the sensitivity of the BL-NAAs using a sensing parameter, that is, changes in the effective optical thickness of the film $(\triangle E O T)$. Our analysis revealed that $\mathrm{BL}-\mathrm{NAA}_{(50 / 50)}$ has the most sensitive sensing pattern amongst BL-NAAs platforms. However, in comparison between a top and bottom structure, the bottom layer with less thickness features higher sensitivity. These results indicate that all these three layers can be used independently for sensing which means that with different surface chemistries this platform can be used for multi analyte biosensing. We believe these types of bilayered NAA structures, with further development of selective chemistry inside the pores, are a promising platform for the footprint development of multi-point sensing RIfS devices for analysis of complex analyte systems including biological and environmental samples.

Supplementary Materials: The following are available online at www.mdpi.com/1424-8220/18/2/470/s1, Fabrication and optimization of nanoporous anodic alumina structures as multi-point interferometric sensing platform.

Acknowledgments: Authors thank the support provided by the Australian Research Council (ARC) Grants FT110100711, DE140100549, the University of Adelaide and the School of Chemical Engineering (UoA).

Author Contributions: The work presented in this paper was a collaboration of all authors. M.N. fabricated all materials, the device, performed all characterizations, data processing. A.S. conceived and designed the experiments; the results were interpreted and the paper was written by M.N. and D.L. Final manuscript was written by M.N. and discussed and approved with all authors.

Conflicts of Interest: The authors declare no conflict of interest.

\section{References}

1. Md Jani, A.M.; Losic, D.; Voelcker, N.H. Nanoporous anodic aluminium oxide: Advances in surface engineering and emerging applications. Prog. Mater. Sci. 2013, 58, 636-704. [CrossRef]

2. Santos, A.; Kumeria, T.; Losic, D. Nanoporous anodic alumina: A versatile platform for optical biosensors. Materials 2014, 7, 4297-4320. [CrossRef] [PubMed]

3. Sheng, X.; Liu, J.; Coronel, N.; Agarwal, A.M.; Michel, J.; Kimerling, L.C. Integration of self-assembled porous alumina and distributed bragg reflector for light trapping in Si photovoltaic devices. IEEE Photon. Technol. Lett. 2010, 22, 1394-1396. [CrossRef]

4. Toccafondi, C.; Thorat, S.; Rocca, R.L.; Scarpellini, A.; Salerno, M.; Dante, S.; Das, G. Multifunctional substrates of thin porous alumina for cell biosensors. J. Mater. Sci. Mater. Med. 2014, 25, 2411-2420. [CrossRef] [PubMed]

5. Yamaguchi, A.; Hotta, K.; Teramae, N. Optical waveguide sensor based on a porous anodic alumina/aluminum multilayer film. Anal. Chem. 2009, 81, 105-111. [CrossRef] [PubMed]

6. Sulka, G.D.; Hnida, K. Distributed bragg reflector based on porous anodic alumina fabricated by pulse anodization. Nanotechnol 2012, 23, 075303. [CrossRef] [PubMed]

7. Rahman, M.M.; Marsal, L.F.; Pallarès, J.; Ferré-Borrull, J. Tuning the photonic stop bands of nanoporous anodic alumina-based distributed bragg reflectors by pore widening. ACS Appl. Mater. Interfaces 2013, 5, 13375-13381. [CrossRef] [PubMed]

8. Dronov, R.; Jane, A.; Shapter, J.G.; Hodges, A.; Voelcker, N.H. Nanoporous alumina-based interferometric transducers ennobled. Nanoscale 2011, 3, 3109-3114. [CrossRef] [PubMed] 
9. Brecht, A.; Gauglitz, G. Recent developments in optical transducers for chemical or biomedical applications. Sens. Actuators B 1997, 38, 1-7. [CrossRef]

10. Reichl, D.; Krage, R.; Krumme, C.; Gauglitz, G. Sensing of volatile organic compounds using a simplified reflectometric interference spectroscopy set up. Appl. Spectrosc. 2000, 54, 583-586. [CrossRef]

11. Belmont, A.-S.; Jaeger, S.; Knopp, D.; Niessner, R.; Gauglitz, G.; Haupt, K. Molecularly imprinted polymer films for reflectometric interference spectroscopic sensors. Biosens. Bioelectron. 2007, 22, 3267-3272. [CrossRef] [PubMed]

12. Leopold, N.; Busche, S.; Gauglitz, G.; Lendl, B. IR absorption and reflectometric interference spectroscopy (RIfS) combined to a new sensing approach for gas analytes absorbed into thin polymer films. Spectrochim. Acta A Mol. Biomol. Spectrosc. 2009, 72, 994-999. [CrossRef] [PubMed]

13. Nemati, M.; Santos, A.; Kumeria, T.; Losic, D. Label-free real-time quantification of enzyme levels by interferometric spectroscopy combined with gelatin-modified nanoporous anodic alumina photonic films. Anal. Chem. 2015, 87, 9016-9024. [CrossRef] [PubMed]

14. Brecht, A.; Gauglitz, G. Optical probes and transducers. Biosens. Bioelectron. 1995, 10, 923-936. [CrossRef] [PubMed]

15. Gauglitz, G. Direct optical sensors: Principles and selected applications. Anal. Bioanal. Chem. 2005, 381, 141-155. [CrossRef] [PubMed]

16. Gauglitz, G. Direct optical detection in bioanalysis: An update. Anal. Bioanal. Chem. 2010, 398, $2363-2372$. [CrossRef] [PubMed]

17. Dancil, K.-P.S.; Greiner, D.P.; Sailor, M.J. A porous silicon optical biosensor: Detection of reversible binding of IgG to a protein A-modified surface. J. Am. Chem. Soc. 1999, 121, 7925-7930. [CrossRef]

18. Kumeria, T.; Santos, A.; Losic, D. Nanoporous Anodic Alumina Platforms: Engineered Surface Chemistry and Structure for Optical Sensing Applications. Sensors 2014, 14, 11878-11918. [CrossRef] [PubMed]

19. Nemati, M.; Santos, A.; Law, C.S.; Losic, D. Assessment of binding affinity between drugs and human serum albumin using nanoporous anodic alumina photonic crystals. Anal. Chem. 2016, 88, 5971-5980. [CrossRef] [PubMed]

20. Santos, A.; Balderrama, V.; Alba, M.; Formentín, P.; Ferré- Borrull, J.; Pallarès, J.; Marsal, L.F. Nanoporous anodic alumina barcodes: Toward smart optical biosensors. Adv. Mater. 2012, 24, 1050-1054. [CrossRef] [PubMed]

21. Kumeria, T.; Kurkuri, M.D.; Diener, K.R.; Parkinson, L.; Losic, D. Label-free reflectometric interference microchip biosensor based on nanoporous alumina for detection of circulating tumor cells. Biosens. Bioelectron. 2012, 35, 167-173. [CrossRef] [PubMed]

22. Kumeria, T.; Parkinson, L.; Losic, D. A nanoporous interferometric micro-sensor for biomedical detection of volatile Sulphur compounds. Nanoscale Res. Lett. 2011, 6, 634. [CrossRef] [PubMed]

23. Law, C.S.; Santos, A.; Nemati, M.; Losic, D. Structural engineering of nanoporous anodic alumina photonic crystals by sawtooth-like pulse anodization. ACS Appl. Mater. Interfaces 2016, 8, 13542-13554. [CrossRef] [PubMed]

24. Law, C.S.; Sylvia, G.M.; Nemati, M.; Yu, J.; Losic, D.; Abell, A.D.; Santos, A. Engineering of surface chemistry for enhanced sensitivity in nanoporous interferometric sensing platforms. ACS Appl. Mater. Interfaces 2017, 9, 8929-8940. [CrossRef] [PubMed]

25. Losic, D.; Lillo, M.; Losci, D., Jr. Porous alumina with shaped pore geometries and complex pore architectures fabricated by cyclic anodization. Small 2009, 5, 1392-1397. [CrossRef] [PubMed]

26. Losic, D.; Losci, D., Jr. preparation of porous anodic alumina with periodically perforated pores. Langmuir 2009, 25, 5426-5431. [CrossRef] [PubMed]

27. Kumeria, T.; Rahman, M.M.; Santos, A.; Ferré-Borrull, J.; Marsal, F.L.; Losic, D. Structural and optical nanoengineering of nanoporous anodic alumina rugate filters for real-time and label-free biosensing applications. Anal. Chem. 2014, 86, 1837-1844. [CrossRef] [PubMed]

28. Macias, G.; Ferré-Borrull, J.; Pallares, J.; Marsal, L.F. 1-D nanoporous anodic alumina rugate filters by means of small current variations for real-time sensing applications. Nanoscale Res. Lett. 2014, 9, 315. [CrossRef] [PubMed]

29. Zheng, W.J.; Fei, G.T.; Wang, B. Modulation of transmission spectra of anodized alumina membrane distributed Bragg reflector by controlling anodization temperature. Nanoscale Res. Lett. 2009, 4, 665-667. [CrossRef] [PubMed] 
30. Santos, A.; Formentín, P.; Pallarès, J.; Ferré-Borrull, J.; Marsal, L.F. Structural engineering of nanoporous anodic alumina funnels with high aspect ratio. J. Electroanal. Chem. 2011, 655, 73-78. [CrossRef]

31. Macias, G.; Hernández-Eguía, L.P.; Ferreé-Borrull, J.; Pallares, J.; Marsal, L.F. Gold-coated ordered nanoporous anodic alumina bilayers for future label-free interferometric biosensors. ACS Appl. Mater. Interfaces 2013, 5, 8093-8098. [CrossRef] [PubMed]

32. Santos, A.; Kumeria, T.; Wang, Y.; Losic, D. In situ monitoring engineering of inverted nanoporous anodic alumina funnels: On the precise generation of 3D optical nanostructures. Nanoscale 2014, 6, 9991-9999. [CrossRef] [PubMed]

33. Wang, H.; Wang, G. Enhanced the optical transmission efficiency by funnel-shaped nanopore. In Proceedings of the International Conference on Manipulation, Manufacturing and Measurement on the Nanoscale (3M-NANO), Chongqing, China, 18-22 July 2016; pp. 55-58. [CrossRef]

34. Manach, C.; Scalbert, A.; Morand, C.; Rémécy, C.; Jiménez, L. Polyphenols: Food sources and bioavailability. Am. J. Clin. Nutr. 2004, 79, 727-747. [CrossRef] [PubMed]

35. Burda, S.; Oleszek, W. Antioxidant and antiradical activities of flavonoids. J. Agric. Food Chem. 2001, 49, 2774-2779. [CrossRef] [PubMed]

36. Wang, J.; Zhou, N.; Zhu, Z.; Huang, J.; Li, G. Detection of flavonoids and assay for their antioxidant activity based on enlargement of gold nanoparticles. Anal. Bioanal. Chem. 2007, 388, 1199-1205. [CrossRef] [PubMed]

37. Lamson, D.W.; Brignall, M.S. Antioxidants and cancer III: Quercetin. Altern Med. Rev. 2000, 5, $196-208$. [PubMed]

38. Jenkins, D.J.A.; Kendall, C.W.; Ransom, T.P.P. Dietary fiber, the evolution of the human diet and coronary heart disease. Nutr. Res. 1998, 18, 633-652. [CrossRef]

39. Mukhopadhyay, M.; Singh, A.; Sachchidanand, S.; Berta, A.K. Quercetin inhibits acid-sensing ion channels through a putative binding site in the central vestibular region. Neuroscience 2017, 348, 264-272. [CrossRef] [PubMed]

40. Velleman, L.; Triani, G.; Evans, P.J.; Shapter, J.G.; Losic, D. Structural and chemical modification of porous alumina membranes. Micropor. Mesopor. Mater. 2009, 126, 87-94. [CrossRef]

41. Kumeria, T.; Losic, D. Controlling interferometric properties of nanoporous anodic aluminium oxide. Nanoscale Res. Lett. 2012, 7, 88. [CrossRef] [PubMed] 